\title{
EXISTENCE AND UNIQUENESS OF POSITIVE SOLUTIONS TO ELLIPTIC PROBLEMS WITH SUBLINEAR MIXED BOUNDARY CONDITIONS*
}

\author{
Jorge García-Melián ${ }^{1}$, Julio D. Rossi ${ }^{2,3}$ and José C. Sabina de $\operatorname{Lis}^{1} \dagger$ \\ 1 Dpto. de Análisis Matemático, Universidad de La Laguna, \\ C/ Astrofísico Francisco Sánchez s/n, 38271 - La Laguna, SPAIN \\ ${ }^{2}$ Facultad de Ciencias Exactas y Naturales, Universidad de Buenos Aires, \\ 1428 - Buenos Aires, ARGENTINA \\ ${ }^{3}$ Instituto de Matemáticas y Física Fundamental, CSIC \\ C/ Serrano 123, 28006 - Madrid, SPAIN
}

\begin{abstract}
In this work we consider a class of semilinear elliptic problems with nonlinear boundary conditions of mixed type. Under some monotonicity properties of the nonlinearities involved, we show that positive solutions are unique, and that their existence is characterized by the sign of some associated eigenvalues. One of the most important contributions of this work relies in the fact that we deal with boundary conditions of the form $\partial u / \partial \nu=g(x, u)$ on $\Gamma$ and $u=0$ on $\Gamma^{\prime}$, where $\nu$ is the outward unit normal to $\Gamma$ while $\Gamma, \Gamma^{\prime}$ are open, $\Gamma \cap \Gamma^{\prime}=\emptyset, \partial \Omega=\bar{\Gamma} \cup \overline{\Gamma^{\prime}}$, but $\bar{\Gamma}, \overline{\Gamma^{\prime}}$ need not be disjoint.
\end{abstract}

\section{INTRODUCTION AND RESULTS}

In a nice and nowadays classical paper $([10])$, Brezis and Oswald introduced an elegant variational approach to study positive solutions to the semilinear problem

$$
\left\{\begin{array}{c}
-\Delta u=f(x, u) \quad x \in \Omega, \\
u_{\mid \partial \Omega}=0 .
\end{array}\right.
$$

Moreover, they provided necessary and sufficient conditions for the existence and uniqueness of solutions, conditions which are solely expressed in terms of the principal eigenvalues of certain natural associated eigenvalue problems.

We are dealing in this paper with a more general kind of boundary value problems, where the nonlinear regime induced by the reaction term $f(x, u)$ is coupled to Dirichlet and

*Supported by MEC and FEDER under grant MTM2005-06480 and ANPCyT PICT No. 03-05009 (J. D. Rossi). J.D. Rossi is a member of CONICET.

†E-mail addresses: jjgarmel@ull.es, jrossi@dm.uba.ar, josabina@ull.es 
semilinear Robin-type conditions, mixed in a nontrivial way on the boundary. Namely,

$$
\begin{cases}-\Delta u=f(x, u) & \text { in } \quad \Omega, \\ \frac{\partial u}{\partial \nu}=g(x, u) & \text { on } \quad \Gamma \\ u=0 & \text { on } \Gamma^{\prime}\end{cases}
$$

where $\Omega$ is a $C^{2, \alpha}$ bounded domain of $\mathbb{R}^{N}, \Gamma, \Gamma^{\prime} \subset \partial \Omega$ disjoint and open, $\bar{\Gamma}, \overline{\Gamma^{\prime}}$ compact manifolds with a common $N-2$ dimensional closed boundary $\gamma=\bar{\Gamma} \cap \overline{\Gamma^{\prime}}$ and $\partial \Omega=\Gamma \cup \gamma \cup \Gamma^{\prime}$.

The subject of elliptic problems constrained by nonlinear flux conditions has been extensively studied since the pioneering works [5], [6], where the method of sub and super solutions was introduced to this pursuit (see also [26]). Since then, the main nonlinear theory issues have been already explored in the topic: uniqueness and multiplicity of positive solutions, stability of equilibria and blow-up of solutions to the associated parabolic problem, and bifurcation driven by the boundary conditions, to quote only a few of them (see [28] for a recent and comprehensive review). However, diffusion problems under mixed boundary conditions, one of them being of nonlinear flux type, have been barely touched in the literature. Precisely, one of the relevant features of the present work is that we are mainly dealing with the case where $\gamma \neq \emptyset$ and so the components $\Gamma$ and $\Gamma^{\prime}$ meet each other. It is well-known that this drives a dramatic loss in the smoothness of solutions, even in the weak sense and regarding the framework of plain linear equations (see for instance [20, [22, [23] and specially [9]). On the other hand, those few works dealing with nonlinear boundary conditions in mixed regime are restricted to impose different conditions on separated components of the boundary (see pioneering results in [27], [26] and more recently [11], [25]). To the best of our knowledge, the only results considered so far for nonlinear equations with genuinely mixed boundary conditions were restricted to Dirichlet-Neumann conditions (see [1], [2], [3], [13], [14] and [15]).

As auxiliary tools, we are also analyzing several types of qualitative properties of two different kind of linear eigenvalue problems. Namely, (1.5), (1.9), the latter being of the Steklov-type (see [6]). By the regularity reasons already mentioned, another of the distinctive achievements of this work is the discussion of such properties under actual mixed conditions. In fact, we are also extending similar results obtained in [12] for mixed problems of the type (1.5), where different boundary conditions are defined in connected pieces of $\partial \Omega$ separated away each other.

With respect to the nonlinear terms $f, g$, we are assuming that $f: \Omega \times[0, \infty) \rightarrow \mathbb{R}$, $g: \partial \Omega \times[0, \infty) \rightarrow \mathbb{R}$ are Carathéodory functions, i.e., $f(x, \cdot)$ (respectively, $g(x, \cdot))$ is continuos in $[0, \infty)$ for almost all $x \in \Omega(\partial \Omega), f(\cdot, u)(g(\cdot, u))$ measurable in $\Omega(\partial \Omega)$ for all $u \in[0, \infty)$, being both $f(\cdot, u)$ and $g(\cdot, u)$ essentially bounded functions for every $u \geq 0$. Moreover, they are sublinear in the sense that

$$
f(x, u) \leq C(1+u) \quad x \in \bar{\Omega}, u \geq 0, \quad g(x, u) \leq C(1+u) \quad x \in \partial \Omega, u \geq 0,
$$

for some positive constant $C$. Finally, we require a monotonicity assumption:

$$
\frac{f(x, u)}{u} \text { and } \frac{g(x, u)}{u} \text { are both decreasing in } u>0 .
$$


These hypotheses will be termed altogether as hypotheses $(\mathrm{H})$. From the continuity of $f(x, u)$ and $g(x, u)$ in $u \geq 0$ it is also implicit in (1.4) that $f(x, 0) \geq 0, g(x, 0) \geq 0$ a. e. in $\Omega$.

As we have mentioned before, an essential part in the issue of existence of solutions to (1.2) will be played by some related eigenvalue problems. Specifically, we need to consider the problems

$$
\begin{cases}-\Delta u=a(x) u+\sigma u & \text { in } \quad \Omega, \\ \frac{\partial u}{\partial \nu}=b(x) u & \text { on } \Gamma, \\ u=0 & \text { on } \Gamma^{\prime},\end{cases}
$$

where $a(x), b(x)$ are measurable functions, not necessarily bounded. Thus, we are defining the principal eigenvalue of (1.5) by means of the usual variational characterization

$$
\sigma_{1}(a, b)=\inf _{\substack{u \in H^{\prime}(\Omega) \\ u \neq 0}} \frac{\int_{\Omega}|\nabla u|^{2}-\int_{\Omega} a(x) u^{2}-\int_{\Gamma} b(x) u^{2}}{\int_{\Omega} u^{2}},
$$

where $H_{\Gamma^{\prime}}^{1}(\Omega)$ stands for the subspace of $H^{1}(\Omega)$ consisting in those functions vanishing on $\Gamma^{\prime}$ in the sense of traces.

If $a$ and $b$ are bounded from above, then $\sigma_{1}(a, b)>-\infty$, but $\sigma_{1}(a, b)=\infty$ is possible. Similarly, when $a$ and $b$ are bounded from below, $\sigma_{1}(a, b)<\infty$, but it could happen that $\sigma_{1}(a, b)=-\infty$. On the other hand, when $a$ and $b$ are bounded $\sigma_{1}(a, b)$ is always finite and possesses an associated positive eigenfunction, together with the usual properties of principal eigenvalues, as isolation, uniqueness and simplicity (see Lemma 7 in Section 2).

To state our results, let us introduce the functions

$$
\begin{aligned}
f_{0}(x) & =\lim _{u \rightarrow 0+} \frac{f(x, u)}{u}, & f_{\infty}(x) & =\lim _{u \rightarrow+\infty} \frac{f(x, u)}{u}, \\
g_{0}(x) & =\lim _{u \rightarrow 0+} \frac{g(x, u)}{u}, & g_{\infty}(x) & =\lim _{u \rightarrow+\infty} \frac{g(x, u)}{u} .
\end{aligned}
$$

Our hypotheses on $f$ and $g$ imply that $f_{\infty}$ and $g_{\infty}$ are bounded from above, while $f_{0}$ and $g_{0}$ are bounded from below. Thus $-\infty<\sigma_{1}\left(f_{\infty}, g_{\infty}\right) \leq+\infty,-\infty \leq \sigma_{1}\left(f_{0}, g_{0}\right)<+\infty$.

Theorem 1. Assume the functions $f(x, u)$ and $g(x, u)$ verify hypotheses $(\mathrm{H})$. Then problem (1.2) has a positive weak solution $u \in H_{\Gamma^{\prime}}^{1}(\Omega) \cap L^{\infty}(\Omega)$ if and only if

$$
\sigma_{1}\left(f_{\infty}, g_{\infty}\right)>0 \quad \text { and } \quad \sigma_{1}\left(f_{0}, g_{0}\right)<0 .
$$

In this case, the positive solution $u$ is unique.

Remarks 1.

a) The monotonicity condition (1.4) is not needed for the existence of solutions (see Lemma 12). However, it is essential for uniqueness (see Lemma 13). On the other hand the normal derivative operator $\frac{\partial}{\partial \nu}$ in $(1.2)$ can be replaced by a Robin operator $\frac{\partial}{\partial \nu}+b_{1}(x), b_{1} \in L^{\infty}(\Gamma)$. This only amounts to shift by $-b_{1}$ the limits $f_{0}, g_{0}, f_{\infty}, g_{\infty}$ in the statement of Theorem 1 . 
b) Some more general operators may be considered instead of the Laplacian, as long as they have a variational structure. For instance, the problem

$$
\begin{cases}-\sum_{i, j=1}^{N} \frac{\partial}{\partial x_{i}}\left(a_{i j}(x) \frac{\partial u}{\partial x_{j}}\right)=f(x, u) & \text { in } \quad \Omega, \\ \sum_{i, j=1}^{N} a_{i j}(x) \frac{\partial u}{\partial x_{j}} \nu_{i}=g(x, u) & \text { on } \Gamma \\ u=0 & \text { on } \Gamma^{\prime}\end{cases}
$$

possesses the same features as (1.2), provided $a_{i j}$ are bounded measurable coefficients which verify the ellipticity condition

$$
\sum_{i, j=1}^{N} a_{i j}(x) \xi_{i} \xi_{j} \geq c|\xi|^{2},
$$

for all $x \in \bar{\Omega}, \xi \in \mathbb{R}^{N}$ and some $c>0$.

The condition (1.8) for existence of solutions can be equivalently stated in terms of the first eigenvalue $\tilde{\sigma}_{1}(a, b)$ to the Steklov-type eigenvalue problem,

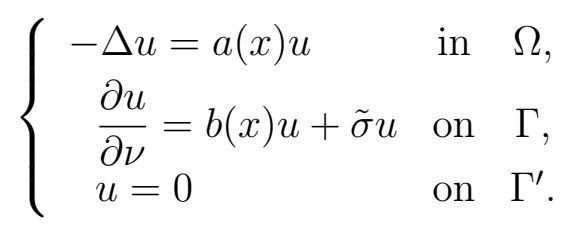

Such eigenvalue is well defined if and only if the first Dirichlet eigenvalue $\lambda_{1}(-a)$ of $-\Delta-a(x)$ in $\Omega$ is positive. In that case $\tilde{\sigma}_{1}(a, b)$ has a variational characterization similar to (1.6) (cf. Lemma 9). Nevertheless, by defining $\tilde{\sigma}_{1}(a, b)$ appropriately when $\lambda_{1}(-a) \leq 0$ (see Lemma 9 and Remarks 2 ), the conclusions of Theorem 1 can be equivalently expressed as follows.

Corollary 2. Under the hypotheses of Theorem 1 problem (1.2) admits a positive solution $u \in H_{\Gamma^{\prime}}^{1}(\Omega)$ if and only if

$$
\lambda_{1}\left(-f_{\infty}\right)>0, \quad \tilde{\sigma}_{1}\left(f_{\infty}, g_{\infty}\right)>0 \quad \text { and } \quad \tilde{\sigma}_{1}\left(f_{0}, g_{0}\right)<0 .
$$

In that case the positive solution is unique.

Among other applications (see Section 4) we are specializing our results to study some classes of logistic-type problems. To describe one of them, we are characterizing the regime of existence (and uniqueness) of positive solutions to

$$
\begin{cases}-\Delta u=\lambda u-a(x) u^{p} & \text { in } \quad \Omega, \\ \frac{\partial u}{\partial \nu}=\mu u-b(x) u^{q} & \text { on } \Gamma, \\ u=0 & \text { on } \Gamma^{\prime}\end{cases}
$$

$p, q>1$, with respect to the parameters $\lambda, \mu \in \mathbb{R}$. Here $a, b$ are nonnegative continuous functions whose zero sets are defined by $\bar{\Omega}_{0}, \bar{\Gamma}_{0}$, respectively, where $\Omega_{0} \subset \Omega$ is a smooth 

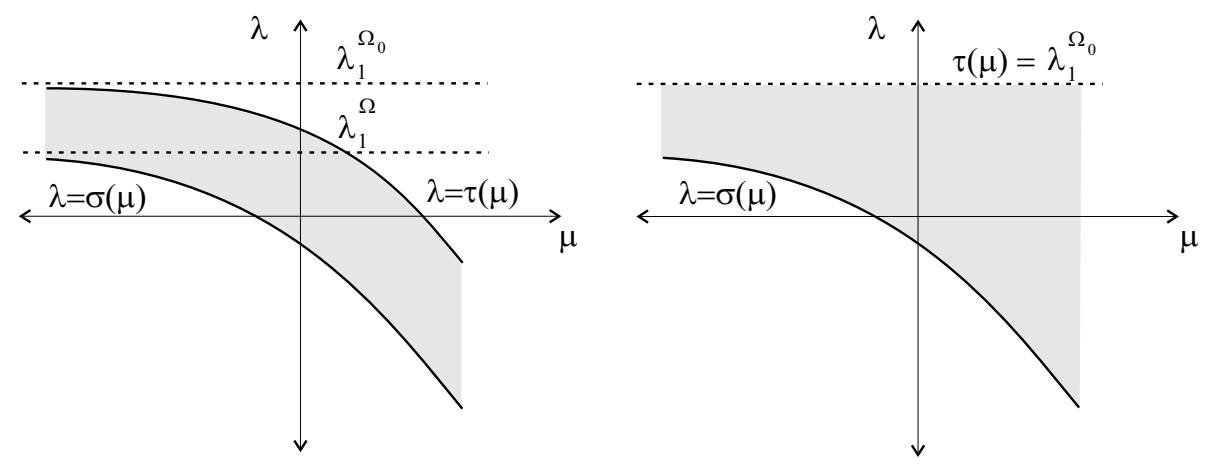

Figure 1: Regions of existence and uniqueness of positive solutions to problem (1.11) corresponding to the cases $\Gamma_{1} \neq \emptyset$ (left) and $\Gamma_{1}=\emptyset$ (right).

domain and $\Gamma_{0} \subset \Gamma$ is open in $\partial \Omega$ so that $\bar{\Gamma}_{0}$ defines a smooth $N-1$ dimensional manifold with boundary. Aside the mixed conditions, it should be remarked that our approach here permits us to handle the case where the null set $\Omega_{0}$ has nontrivial intersection with both the Dirichlet component $\Gamma^{\prime}$, and the component $\Gamma$ supporting the flux condition. In fact, such a contact has been prohibited even for logistic problems with a single linear Robin condition (see [16] and Section 4). In this sense, we improve the corresponding results in [11], [12]. Such improvement also comprises the response of the principal eigenvalues of the problems (1.5), (1.9) in the presence of singular weights $a, b$ (see Lemma 10).

It turns out that the relevant eigenvalues $\sigma_{1}\left(f_{0}, g_{0}\right), \sigma_{1}\left(f_{\infty}, g_{\infty}\right)$ corresponding to (1.11) can be characterized in terms of the principal eigenvalues $\lambda=\sigma(\mu)$ and $\lambda=\tau(\mu)$ of the problems

$$
\left\{\begin{array} { l l } 
{ - \Delta u = \lambda u } & { \text { in } \quad \Omega , } \\
{ \frac { \partial u } { \partial \nu } = \mu u } & { \text { on } \Gamma , } \\
{ u = 0 } & { \text { on } \Gamma ^ { \prime } , }
\end{array} \quad \text { and } \quad \left\{\begin{array}{lll}
-\Delta u=\lambda u & \text { in } \Omega_{0}, \\
\frac{\partial u}{\partial \nu}=\mu u & \text { on } \Gamma_{1}, \\
u=0 & \text { on } \Gamma_{2},
\end{array}\right.\right.
$$

respectively, where $\Gamma_{1}:=\partial \Omega_{0} \cap \Gamma_{0}, \Gamma_{2}:=\partial \Omega_{0} \backslash \bar{\Gamma}_{1}$ are open manifolds. For the sake of simplicity it is additionally assumed (cf. Lemma 10 for more precise requirements on $\partial \Omega_{0}$ ) that $\bar{\Gamma}_{1}$ is either a smooth submanifold of $\partial \Omega$ with boundary or $\bar{\Gamma}_{1}=\emptyset$, being $\tau(\mu) \equiv \lambda_{1}^{\Omega_{0}}$ in the latter case, where $\lambda_{1}^{\Omega_{0}}$ stands for the first Dirichlet eigenvalue of $-\Delta$ in $\Omega_{0}$. These problems will be analyzed in detail in Section 2 (cf. Lemma 8 ). Observe that according to (1.5), $\sigma(\mu)=\sigma_{1}(a, b)$ with $a=0, b=\mu$.

Our results concerning problem (1.11) reads as follows (see Figure 1):

Theorem 3. Assume $p, q>1$. Then problem (1.11) admits a positive weak solution $u \in$ $H_{\Gamma^{\prime}}^{1}(\Omega) \cap L^{\infty}(\Omega)$ if and only if $\sigma(\mu)<\lambda<\tau(\mu)$. In that case, the solution $u$ is unique.

It should be noticed that $\sigma_{1}(\mu)<\tau(\mu)$ for every $\mu \in \mathbb{R}$ (so the region introduced in Theorem 3 is nonempty), $\lim \sigma(\mu)=\lim \tau(\mu)=-\infty$ as $\mu \rightarrow \infty$, while $\sigma(\mu) \rightarrow \lambda_{1}^{\Omega}$, $\tau(\mu) \rightarrow \lambda_{1}^{\Omega_{0}}$ as $\mu \rightarrow-\infty$ (Figure 1). See Lemma 8 and the proof of Theorem 3 .

On the other hand, the eigenvalue problems (1.12) admit an Steklov reading instead of the "volumetric" one observed above. In fact, by regarding now $\lambda$ as a parameter and $\mu$ as an eigenvalue, the first problem in (1.12) defines the principal Steklov eigenvalue $\mu=\tilde{\sigma}(\lambda)$ 

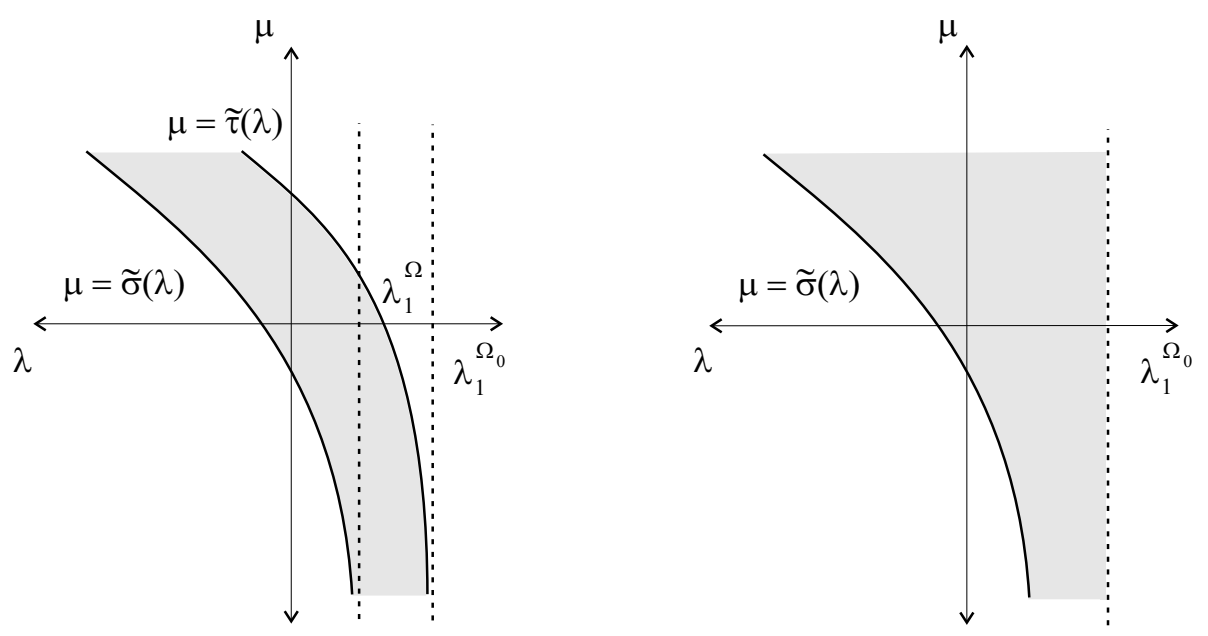

Figure 2: Regions of existence and uniqueness of positive solutions to problem (1.11) expressed in terms of Stleklov eigenvalues $\left(\Gamma_{1} \neq \emptyset\right.$ on the left, the figure on the right corresponding to $\left.\Gamma_{1}=\emptyset\right)$.

as a function of $\lambda$, the same being true for the second one expressing its principal eigenvalue $\mu=\tilde{\tau}(\lambda)$. Accordingly, Theorem 3 admits the following dual statement.

Corollary 4. In the conditions of Theorem 3, problem (1.11) admits a positive weak solution if and only if $\lambda<\lambda_{1}^{\Omega_{0}}$ and either

$$
\tilde{\sigma}(\lambda)<\mu<\tilde{\tau}(\lambda)
$$

if $\Gamma_{1} \neq \emptyset$ or

$$
\tilde{\sigma}(\lambda)<\mu,
$$

when $\Gamma_{1}=\emptyset$, where $\tilde{\sigma}(\lambda)=-\infty$ for $\lambda \geq \lambda_{1}^{\Omega}$. Such solution is unique (Figure 2).

The paper is organized as follows: Section 2 is devoted to some preliminary facts, namely the boundedness of weak solutions to (1.2) and the analysis of the eigenvalue problems (1.5) and (1.12). In Section 3 we prove Theorem 1 while applications to some nonlinear problems, which include a proof of Theorem 3, are presented in Section 4.

\section{Preliminaries}

In this section some preliminary results needed for our subsequent developments are collected. We begin with some remarks concerning the definition of weak solution to our problem. A nonnegative function $u \in H_{\Gamma^{\prime}}^{1}(\Omega)$ is a weak solution to (1.2) provided that, under the hypotheses $(\mathrm{H}), f(\cdot, u(\cdot)) \in L^{2}(\Omega), g(\cdot, u(\cdot)) \in L^{2}(\partial \Omega)$ and

$$
\int_{\Omega}\{\nabla u \nabla \varphi-f(x, u) \varphi\}-\int_{\partial \Omega} g(x, u) \varphi=0,
$$

for every $\varphi \in H_{\Gamma^{\prime}}^{1}(\Omega)$. The existence of this kind of solutions to (1.2) will be obtained in Section 3 were the extra integrability conditions on $f(\cdot, u(\cdot)), g(\cdot, u(\cdot))$ follow once one is able to prove that the candidate to solution $u \in H_{\Gamma^{\prime}}^{1}(\Omega)$ indeed belongs to $L^{\infty}(\Omega)$. 
In order to achieve in Section 3 such boundedness result, we are first dealing with the more restrictive class of problems were the growth conditions (1.3) are replaced by

$$
|f(x, u)| \leq C(1+u) \quad x \in \bar{\Omega}, u \geq 0, \quad|g(x, u)| \leq C(1+u) \quad x \in \partial \Omega, u \geq 0 .
$$

For such problems the definition (2.1) of a weak solution $u \in H_{\Gamma^{\prime}}^{1}(\Omega)$ has full sense without additional requirements on $f(\cdot, u(\cdot)), g(\cdot, u(\cdot))$. We also remark that under $(2.2)$ and by interior regularity (see [20]), nonnegative weak solutions $u \in H_{\Gamma^{\prime}}^{1}(\Omega)$ also belong to $W_{\text {loc }}^{2, p}(\Omega)$ for every $p>1$, and hence $u \in C_{\text {loc }}^{1, \alpha}(\Omega)$ for every $0<\alpha<1$. Moreover, since for each subdomain $\Omega^{\prime} \subset \overline{\Omega^{\prime}} \subset \Omega$ a positive $M$ exists so that $f(x, u) \geq-M u$ (condition (1.4)), it follows that $-\Delta u+M u \geq 0$ in $\Omega^{\prime}$, and the maximum principle implies that $u>0$ in $\Omega^{\prime}$, unless $u$ is the trivial solution. Hence, either $u>0$ in $\Omega$ or $u \equiv 0$.

We are next proving that -under the growth conditions $(2.2)$ - positive weak solutions to (1.2) are essentially bounded. In addition to the existence issue already mentioned, this fact will be also essential in order to achieve uniqueness of solutions. The proof relies on Moser's iteration procedure (see [20]).

Lemma 5. Let $u \in H_{\Gamma^{\prime}}^{1}(\Omega)$ be a weak solution to (1.2) and assume $f$ and $g$ verify hypotheses $(\mathrm{H})$ with $(1.3)$ replaced by (2.2). Then $u \in L^{\infty}(\Omega)$. Moreover, there exists a positive constant $C$, not depending on $u$, such that

$$
|u|_{L^{\infty}(\Omega)} \leq C\left(|u|_{L^{2}(\Omega)}+1\right)
$$

Proof. Let $\beta \geq 1, k>1$, and take as a test function in the weak formulation (2.1) of problem (1.2):

$$
\varphi=\min \left\{v^{\beta}, k^{\beta}\right\}-1,
$$

where $v=u+1$. It is not hard to show that $\varphi \in H_{\Gamma^{\prime}}^{1}(\Omega)$. Then

$$
\begin{aligned}
\beta \int_{\{v<k\}} v^{\beta-1}|\nabla v|^{2} & =\int_{\Gamma} g(x, u) \varphi+\int_{\Omega} f(x, u) \varphi \\
& \leq C\left(\int_{\Gamma} v \varphi+\int_{\Omega} v \varphi\right) \\
& \leq C\left(\int_{\Gamma} v^{\beta+1}+\int_{\Omega} v^{\beta+1}\right) .
\end{aligned}
$$

Taking into account that

$$
\beta v^{\beta-1}|\nabla v|^{2} \chi_{\{v<k\}}=\frac{4 \beta}{(\beta+1)^{2}}\left|\nabla\left(v^{\frac{\beta+1}{2}}\right)\right|^{2} \chi_{\{v<k\}}=\frac{4 \beta}{(\beta+1)^{2}}|\nabla \psi|^{2},
$$

where $\psi=\min \left\{v^{\frac{\beta+1}{2}}, k^{\frac{\beta+1}{2}}\right\}$, and using the continuity of the embedding $H^{1}(\Omega) \subset L^{r}(\partial \Omega)$ for $1 \leq r \leq 2_{\partial \Omega}^{*}$, where $2_{\partial \Omega}^{*}=2(N-1) /(N-2)$ if $N \geq 3$ and $2_{\partial \Omega}^{*}=\infty$ for $N=2$ (cf. [4]), we obtain

$$
\int_{\Omega}|\nabla \psi|^{2} \geq C\left(\int_{\partial \Omega} \psi^{r}\right)^{\frac{2}{r}}-\int_{\Omega} \psi^{2} \geq C\left(\int_{\Gamma} \psi^{r}\right)^{\frac{2}{r}}-\int_{\Omega} \psi^{2} .
$$


Fix $r$ so that $2<r \leq 2_{\partial \Omega}^{*}$. From $(2.4)$ and $(2.5)$ we have

$$
\frac{4 \beta}{(\beta+1)^{2}}\left(\left(\int_{\Gamma} \psi^{r}\right)^{\frac{2}{r}}-\int_{\Omega} \psi^{2}\right) \leq C\left(\int_{\Gamma} v^{\beta+1}+\int_{\Omega} v^{\beta+1}\right),
$$

and since $\psi^{2} \leq v^{\beta+1}$ and $1 /(\beta+1) \leq 4 \beta /(\beta+1)^{2} \leq 4$, we arrive at

$$
\left(\int_{\Gamma} \min \left\{v^{\frac{(\beta+1) r}{2}}, k^{\frac{(\beta+1) r}{2}}\right\}\right)^{\frac{2}{r}} \leq C(\beta+1)\left(\int_{\Gamma} v^{\beta+1}+\int_{\Omega} v^{\beta+1}\right) .
$$

Assume now that $u \in L^{\beta+1}(\Omega) \cap L^{\beta+1}(\Gamma)$. Then we can let $k \rightarrow+\infty$ in (2.6) to obtain

$$
\left(\int_{\Gamma} v^{\frac{(\beta+1) r}{2}}\right)^{\frac{2}{r}} \leq C(\beta+1)\left(\int_{\Gamma} v^{\beta+1}+\int_{\Omega} v^{\beta+1}\right) .
$$

In the same way, by using in (2.4) the embedding $H^{1}(\Omega) \subset L^{r}(\Omega)$, which is valid for the value of $r$ fixed before (it is indeed valid for $1 \leq r \leq 2_{\Omega}^{*}$, where $2_{\Omega}^{*}=2 N /(N-2)$ if $N \geq 3$ and $2_{\Omega}^{*}=\infty$ for $N=2$ ), and proceeding analogously, we obtain the estimate

$$
\left(\int_{\Omega} v^{\frac{(\beta+1) r}{2}}\right)^{\frac{2}{r}} \leq C(\beta+1)\left(\int_{\Gamma} v^{\beta+1}+\int_{\Omega} v^{\beta+1}\right) .
$$

Adding (2.7) and (2.8), taking into account that $(a+b)^{\frac{2}{r}} \leq a^{\frac{2}{r}}+b^{\frac{2}{r}}$ for $a, b \geq 0$, and raising to the power $1 /(\beta+1)$ we get

$$
\left(\int_{\Gamma} v^{\frac{(\beta+1) r}{2}}+\int_{\Omega} v^{\frac{(\beta+1) r}{2}}\right)^{\frac{2}{(\beta+1) r}} \leq(C(\beta+1))^{\frac{1}{\beta+1}}\left(\int_{\Gamma} v^{\beta+1}+\int_{\Omega} v^{\beta+1}\right)^{\frac{1}{\beta+1}} .
$$

If we introduce the norm

$$
\|v\|_{p}=\left(\int_{\Gamma}|v|^{p}+\int_{\Omega}|v|^{p}\right)^{\frac{1}{p}}
$$

and set $p=\beta+1, q=r / 2>1$, we have shown that if, for some $p \geq 2$ we have $u \in$ $L^{p}(\Omega) \cap L^{p}(\Gamma)$, then $u \in L^{q p}(\Omega) \cap L^{q p}(\Gamma)$, and

$$
\|v\|_{q p} \leq(C p)^{\frac{1}{p}}\|v\|_{p}
$$

for a certain positive $C>0$ not depending on $p$ and $v$. We now take for $p$ in (2.9) the values $p=2 q^{n}, n=0,1, \ldots$ We then obtain:

$$
\|v\|_{2 q^{n}} \leq(2 C)^{1 / 2}(2 q C)^{1 / 2 q} \cdots\left(2 q^{n} C\right)^{1 / 2 q^{n}}\|v\|_{2} .
$$

The constants in the right hand side can be bounded independently of $n$. Indeed

$$
(2 C)^{1 / 2}(2 q C)^{1 / 2 q} \cdots\left(2 q^{n} C\right)^{1 / 2 q^{n}}<\left((2 C)^{\sum_{n=0}^{\infty} \frac{1}{q^{n}}} q^{\sum_{n=1}^{\infty} \frac{n}{q^{n}}}\right)^{1 / 2}=C,
$$

and we have shown in particular that

$$
|v|_{L^{2 q^{n}}(\Omega)} \leq C\left(|v|_{L^{2}(\Omega)}+|v|_{L^{2}(\Gamma)}\right)
$$


where $C$ does not depend on $v$ and $n$. Letting $n \rightarrow+\infty$, we obtain $v \in L^{\infty}(\Omega)$, and

$$
|v|_{L^{\infty}(\Omega)} \leq C\left(|v|_{L^{2}(\Omega)}+|v|_{L^{2}(\Gamma)}\right) .
$$

This finally implies

$$
|u|_{L^{\infty}(\Omega)} \leq C\left(|u|_{L^{2}(\Omega)}+|u|_{L^{2}(\Gamma)}+1\right)
$$

To obtain (2.3), we take $u$ as a test function in (1.2) and obtain:

$$
\int_{\Omega}|\nabla v|^{2} \leq C\left(\int_{\Omega} v^{2}+\int_{\Gamma} v^{2}\right)
$$

On the other hand, by means of the interpolation inequality (2.11) (see Lemma 6 below), we have that

$$
\int_{\Gamma} v^{2} \leq \varepsilon \int_{\Omega}|\nabla v|^{2}+C(\varepsilon) \int_{\Omega} v^{2} \leq C \varepsilon \int_{\Gamma} v^{2}+C(\varepsilon) \int_{\Omega} v^{2}
$$

Choosing $\varepsilon$ small enough, we can estimate the integral on $\Gamma$ in terms of that in $\Omega$, which, together with (2.10) proves (2.3).

We now turn to prove an interpolation inequality which was used in the previous proof, and which is going to be useful in Section 3 when proving Theorem 1. We include a proof for the sake of completeness.

Lemma 6. For every $\varepsilon>0$, there exists a positive constant $C(\varepsilon)$ such that

$$
\int_{\partial \Omega} u^{2} \leq \varepsilon \int_{\Omega}|\nabla u|^{2}+C(\varepsilon) \int_{\Omega} u^{2}
$$

for every $u \in H^{1}(\Omega)$.

Proof. Fix $p \in(1,2)$. Thanks to the continuity of the immersion $W^{1, p}(\Omega) \subset L^{p}(\partial \Omega)$ (cf. [4]), there exists $C=C(p, N, \Omega)$ such that

$$
\int_{\partial \Omega}|v|^{p} \leq C\left(\int_{\Omega}|\nabla v|^{p}+\int_{\Omega}|v|^{p}\right)
$$

for every $v \in W^{1, p}(\Omega)$. Taking $v=|u|^{2 / p}$, where $u \in H^{1}(\Omega)$, we obtain

$$
\int_{\partial \Omega} u^{2} \leq C\left(\int_{\Omega}|u|^{2-p}|\nabla u|^{p}+\int_{\Omega} u^{2}\right)
$$

Using Young's inequality in the form $a b \leq \varepsilon a^{q}+\varepsilon^{-q^{\prime} / q} b^{q^{\prime}}$ with $q=2 / p$, and $a=|\nabla u|^{p}$, $b=C|u|^{2-p}$, we arrive at

$$
\begin{aligned}
\int_{\partial \Omega} u^{2} & \leq \varepsilon \int_{\Omega}|\nabla u|^{2}+C^{\frac{2}{2-p}} \varepsilon^{-q^{\prime} / q} \int_{\Omega} u^{2}+C \int_{\Omega} u^{2} \\
& =\varepsilon \int_{\Omega}|\nabla u|^{2}+C(\varepsilon) \int_{\Omega} u^{2}
\end{aligned}
$$

for every $u \in H^{1}(\Omega)$, as we wanted to prove. 
We come next to consider problem (1.5) when the weights $a(x), b(x)$ are bounded. The content of the next lemma is probably well-known when $\Gamma$ and $\Gamma^{\prime}$ are disjoint connected components of $\partial \Omega$. For the case where Robin-type and Dirichlet conditions are defined in different connected pieces of $\partial \Omega$ we refer to [7], where very general problems are considered; see also [12] where the domains, coefficients and solutions involved are required to be smooth enough and different boundary conditions appear on different components of $\partial \Omega$. The variational approach of our next result together with Lemma 5 allow us to give a direct proof even in the case of "genuinely mixed" boundary conditions. There, the term $\sigma \in \mathbb{R}$ an eigenvalue of (1.5) is understood as the existence of a nontrivial $\phi \in H_{\Gamma^{\prime}}^{1}(\Omega)$ such that,

$$
\int_{\Omega}\{\nabla \phi \nabla v-a \phi v\}-\int_{\Gamma} b \phi v=\sigma \int_{\Omega} \phi v
$$

for all $v \in H_{\Gamma^{\prime}}^{1}(\Omega)$. Additionally, a "principal" eigenvalue means an eigenvalue with a nonnegative associated eigenfunction.

Lemma 7. Assume $a \in L^{\infty}(\Omega), b \in L^{\infty}(\Gamma)$. Then the eigenvalue problem (1.5):

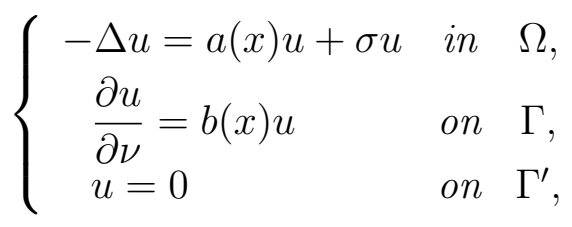

admits a unique principal eigenvalue $\sigma_{1}(a, b)$ given by (1.6). Moreover,

a) $\sigma_{1}(a, b)$ is simple and any associated eigenfunction $\phi$ verifies $\phi \in H_{\Gamma^{\prime}}^{1}(\Omega) \cap L^{\infty}(\Omega)$.

b) $\sigma_{1}(a, b)$ varies monotonically and continuously with respect to $a \in L^{\infty}(\Omega), b \in L^{\infty}(\Gamma)$.

c) Setting $\lambda_{1}(-a)$ the principal Dirichlet eigenvalue of $-\Delta-a$ in $\Omega$ then

$$
\sigma_{1}(a, b)<\lambda_{1}(-a)
$$

for every $b \in L^{\infty}(\Gamma)$.

d) If $\phi \in H_{\Gamma^{\prime}}^{1}(\Omega)$ is any eigenfunction associated to $\sigma_{1}(a, b)$ then $\phi \not \equiv 0$ on $\Gamma$.

Proof. It suffices to prove that the functional

$$
I(u)=\int_{\Omega}|\nabla u|^{2}-\int_{\Omega} a(x) u^{2}-\int_{\Gamma} b(x) u^{2}
$$

attains the infimum in the weakly closed manifold $\mathcal{M}=\left\{u \in H_{\Gamma^{\prime}}^{1}(\Omega): \int_{\Omega} u^{2}=1\right\}$.

We claim that $I$ is coercive. Indeed, thanks to Lemma 6, for $\varepsilon>0$ small, we have that

$$
\int_{\Omega} a(x) u^{2}+\int_{\Gamma} b(x) u^{2} \leq C\left(\int_{\Omega} u^{2}+\int_{\Gamma} u^{2}\right) \leq C(\varepsilon) \int_{\Omega} u^{2}+C \varepsilon \int_{\Omega}|\nabla u|^{2}
$$

where $C$ denotes a positive constant, not necessarily the same everywhere. This implies

$$
I(u) \geq(1-C \varepsilon) \int_{\Omega}|\nabla u|^{2}-C
$$


in $\mathcal{M}$, which in particular shows that $I$ is coercive. Since $I$ is trivially weakly lower semicontinuous, by a well known theorem in the calculus of variations (see [29]) it follows that $I$ attains its infimum in $\mathcal{M}$ at some $\phi \in H_{\Gamma^{\prime}}^{1}(\Omega)$. Since $\phi \neq 0$ it can be assumed, say, that $\phi^{+}=\max \{\phi, 0\}$ is nontrivial. It is then checked that $\phi^{+}$is also an eigenfunction, the maximum principle implies that $\phi^{+}$is positive and thus the same holds for $\phi$ (see [19] for related ideas). The simplicity of $\sigma_{1}(a, b)$ and its uniqueness as a principal eigenvalue can be shown following [17, 18] (cf. also [21]), and we omit the proof.

The fact that $\sigma_{1}\left(a_{1}, b_{1}\right) \geq \sigma_{1}\left(a_{2}, b_{2}\right)$ when $a_{1} \leq a_{2}$ in $L^{\infty}(\Omega)$ and $b_{1} \leq b_{2}$ in $L^{\infty}(\Gamma)$ is an immediate consequence of the variational characterization (1.6). As for the continuity in $a, b$, we include a proof, since it is not straightforward. Beginning with a symmetric case we have for $0<\delta<\delta_{0}$

$$
\sigma_{1}(a, b)>\sigma_{1}(a+\delta, b+\delta) \geq \sigma_{1}(a, b)+O(\delta)
$$

as $\delta \rightarrow 0+$. In fact,

$$
\sigma_{1}(a+\delta, b+\delta)=\int_{\Omega}\left\{\left|\nabla \phi_{\delta}\right|^{2}-a \phi_{\delta}^{2}\right\}-\int_{\Gamma} b \phi_{\delta}^{2}-\delta \int_{\Gamma} \phi_{\delta}^{2}-\delta \geq \sigma_{1}(a, b)-\delta \int_{\Gamma} \phi_{\delta}^{2}-\delta
$$

where $\phi_{\delta} \in H_{\Gamma^{\prime}}^{1}(\Omega)$ stands for the positive eigenfunction associated to $\sigma_{1}(a+\delta, b+\delta)$, normalized as $\int_{\Omega} \phi_{\delta}^{2}=1$. In addition, $\int_{\Gamma} \phi_{\delta}^{2}=O(1)$ as $\delta \rightarrow 0$ since, by comparison, $\sigma_{1}(a+$ $\delta, b+\delta)=O(1)$ and from (2.11) we have the estimate,

$$
\sigma_{1}(a+\delta, b+\delta) \geq(1-\varepsilon) \int_{\Omega}\left|\nabla \phi_{\delta}\right|^{2}-K
$$

for certain $0<\varepsilon<1, K>0$. This yields $\int_{\Omega}\left|\nabla \phi_{\delta}\right|^{2}=O(1)$ which implies $\int_{\Gamma} \phi_{\delta}^{2}=O(1)$ as $\delta \rightarrow 0$. Thus, (2.13) holds.

The complementary behavior $(\delta>0)$

$$
\sigma_{1}(a, b)<\sigma_{1}(a-\delta, b-\delta) \leq \sigma_{1}(a, b)+O(\delta)
$$

is more directly proved and so $\lim _{\delta \rightarrow 0} \sigma_{1}(a+\delta, b+\delta)=\sigma_{1}(a, b)$.

To conclude observe that $a_{n} \rightarrow a, b_{n} \rightarrow b$ means that $a(x)-\delta \leq a_{n}(x) \leq a(x)+\delta$, $b(x)-\delta \leq b_{n}(x) \leq b(x)+\delta$ a.e. in $\Omega$ and $\Gamma$ respectively, for any prefixed $\delta$ and $n$ large. The desired continuity follows by comparison. This proves $\mathrm{b})$.

To show c) observe that $\sigma_{1}(a, b) \leq \lambda_{1}(-a)$ follows by inserting functions $u \in H_{0}^{1}(\Omega)$ in the variational characterization (1.6) of $\sigma_{1}(a, b)$. Thus,

$$
\sigma_{1}(a, b)<\sigma_{1}(a, b-1) \leq \lambda_{1}(-a) .
$$

As desired.

As for d) if a principal eigenfunction $\phi \in H_{\Gamma^{\prime}}^{1}(\Omega)$ vanishes on $\Gamma$ we have that $\phi \in H_{0}^{1}(\Omega)$ and

$$
\sigma_{1}(a, b) \geq \int_{\Omega}|\nabla \phi|^{2}-a \phi^{2} \geq \lambda_{1}(-a)
$$

which contradicts (2.12). 
We are now paying special attention to the behavior of the principal eigenvalue of the problem (1.5) with respect to parameters together with its asymptotic behaviour. Specifically, consider the parametric version of (1.5),

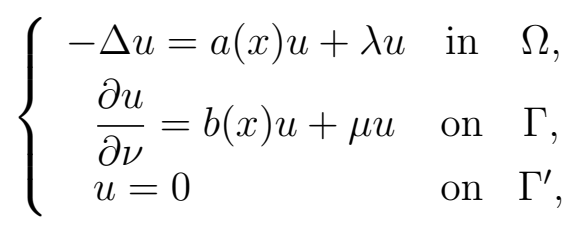

where, for the moment, $\lambda \in \mathbb{R}$ is regarded as an eigenvalue, $\mu$ as a parameter (of course, such rôles can be interchanged), $a \in L^{\infty}(\Omega), b \in L^{\infty}(\Gamma)$. There are two sources of interest in (2.14) regarding the present work. The first is to provide a proper interpretation of the eigenvalue problem (1.9) without restrictions in $a$ (see Lemma 9 below). The second being the analysis of the particular case $a=0, b=0$ (see (1.12) in Section 1 and Theorem 3)

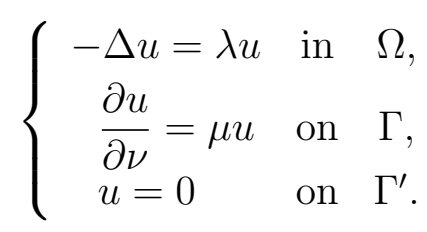

We collect in the next lemma some of the most important features. Regarding (2.15) with $\Gamma^{\prime}=\emptyset$ we refer the reader to [21] for continuity, concavity and the limit (2.16) (as well as some additional asymptotic properties).

Lemma 8. Let $\lambda=\sigma(\mu)$ be the principal eigenvalue of (2.14) for $\mu \in \mathbb{R}$. Then $\sigma(\mu)$ is concave and

$$
\lim _{\mu \rightarrow-\infty} \sigma(\mu)=\lambda_{1}(-a)
$$

while

$$
\lim _{\mu \rightarrow+\infty} \sigma(\mu)=-\infty
$$

Proof. Notice that $\sigma(\mu)=\sigma_{1}(a, b+\mu)$ and thus $\sigma_{1}(\mu)$ is decreasing, $\sigma_{1}(\mu)<\lambda(-a)$ for each $\mu \in \mathbb{R}$ (Lemma 7). By denoting $I_{\mu}(u)=\int_{\Omega}|\nabla u|^{2}-a u^{2}-\int_{\Gamma} b u^{2}-\mu \int_{\Gamma} u^{2}$ we have, for $t \in(0,1)$

$$
\begin{aligned}
\sigma\left(t \mu+(1-t) \mu^{\prime}\right) & =I_{t \mu+(1-t) \mu^{\prime}}\left(\phi_{t \mu+(1-t) \mu^{\prime}}\right) \\
& =t I_{\mu}\left(t \mu+(1-t) \mu^{\prime}\right)+(1-t) I_{\mu^{\prime}}\left(t \mu+(1-t) \mu^{\prime}\right) \\
& \geq t \sigma(\mu)+(1-t) \sigma\left(\mu^{\prime}\right),
\end{aligned}
$$

and the concavity is proved.

The concavity implies that $\sigma(\mu)$ has a derivative a. e. while such derivative must be negative at least at infinitely many values of $\mu$. Thus (2.17) follows.

To prove (2.16) recall that $\sigma(\mu)<\lambda_{1}(-a)$. Choosing $\mu_{n} \rightarrow-\infty$ an arbitrary sequence we have

$$
\sigma\left(\mu_{n}\right)=\int_{\Omega}\left\{\left|\nabla \phi_{\mu_{n}}\right|^{2}-a \phi_{n}^{2}\right\}-\int_{\Gamma} b \phi_{\mu_{n}}^{2}-\mu_{n} \int_{\Gamma} \phi_{\mu_{n}}^{2} \geq \int_{\Omega}\left|\nabla \phi_{\mu_{n}}\right|^{2}-M,
$$


for large enough $n$ and a certain $M>0$. So passing to a subsequence if necessary we obtain $\phi_{\mu_{n}} \rightarrow \phi$ weakly in $H^{1}(\Omega)$, strongly in $L^{2}(\Omega)$ and $L^{2}(\Gamma)$. By taking limits in $(2.18)$ we arrive at

$$
\lambda_{1}(-a) \geq \limsup _{n \rightarrow \infty} \sigma\left(\mu_{n}\right) \geq \liminf _{n \rightarrow \infty} \sigma\left(\mu_{n}\right) \geq \int_{\Omega}|\nabla \phi|^{2}-a \phi^{2} .
$$

Moreover, we also have $\int_{\Omega} \phi^{2}=1$, and

$$
\int_{\Gamma} \phi^{2}=\lim _{n \rightarrow \infty} \int_{\Gamma} \phi_{\mu_{n}}^{2} \leq \lim _{n \rightarrow \infty} \frac{\sigma\left(\mu_{n}\right)+O(1)}{-\mu_{n}}=0 .
$$

This implies $\phi \in H_{0}^{1}(\Omega)$, and by means of the variational characterization of $\lambda_{1}^{\Omega}$ we obtain from $(2.19)$ that

$$
\lim _{n \rightarrow \infty} \sigma\left(\mu_{n}\right)=\lambda_{1}(-a)
$$

Since the sequence $\mu_{n}$ was arbitrary, (2.16) is now proved.

Let us examine now the alternative eigenvalue problem (1.9),

$$
\begin{cases}-\Delta u=a(x) u & \text { in } \quad \Omega, \\ \frac{\partial u}{\partial \nu}=b(x) u+\tilde{\sigma} u & \text { on } \quad \Gamma, \\ u=0 & \text { on } \Gamma^{\prime},\end{cases}
$$

$a \in L^{\infty}(\Omega), b \in L^{\infty}(\Gamma)$. A number $\tilde{\sigma}$ is said to be an eigenvalue of (1.9) if there exists $\psi \in H_{\Gamma^{\prime}}^{1}(\Omega)$ which is nontrivial on $\Gamma$ such that

$$
\int_{\Omega}\{\nabla \psi \nabla v-a \psi v\}-\int_{\Gamma} b \psi v=\tilde{\sigma} \int_{\Gamma} \psi v
$$

for all $v \in H_{\Gamma^{\prime}}^{1}(\Omega)$. The main features concerning (1.9) are collected in the next statement.

Lemma 9. Problem (1.9) admits a principal eigenvalue $\tilde{\sigma}_{1}(a, b)$ if and only if

$$
\lambda_{1}(-a)>0
$$

$\lambda_{1}(-a)$ designating the first Dirichlet eigenvalue of $-\Delta-a$ in $\Omega$. Such eigenvalue is given by,

$$
\tilde{\sigma}_{1}(a, b)=\inf _{\substack{u \in H^{1}(\Omega) \\ u \neq 0}} \frac{\int_{\Omega}|\nabla u|^{2}-\int_{\Omega} a(x) u^{2}-\int_{\Gamma} b(x) u^{2}}{\int_{\Gamma} u^{2}} .
$$

Moreover, the infimum in $(2.20)$ is $-\infty$ provided $\lambda(-a) \leq 0 . \tilde{\sigma}_{1}(a, b)$ is unique, simple and every associated eigenfunction $\phi$ belongs to $H_{\Gamma^{\prime}}^{1}(\Omega) \cap L^{\infty}(\Omega)$. In addition, $\tilde{\sigma}_{1}(a, b)$ exhibits the same continuity and monotonicity properties as $\sigma_{1}(a, b)$ with respect to $a \in L^{\infty}(\Omega), b \in$ $L^{\infty}(\Gamma)$. Finally,

$$
\operatorname{sign} \tilde{\sigma}_{1}(a, b)=\operatorname{sign} \sigma_{1}(a, b)
$$

Remarks 2. 
a) Lemma 9 allows us to define $\tilde{\sigma}_{1}(a, b)$ regardless the sign of $\lambda_{1}(-a)$ by setting $\tilde{\sigma}_{1}(a, b)=-\infty$ when $\lambda_{1}(-a) \leq 0$.

b) As another consequence, Lemma 9 implies that the principal eigenvalue $\mu=\tilde{\sigma}(\lambda):=$ $\sigma_{1}(\lambda, 0)$ is the inverse function of $\sigma(\mu)$ which has the value $-\infty$ as $\lambda \geq \lambda_{1}^{\Omega}$. Moreover $\tilde{\sigma} \rightarrow \infty$ as $\lambda \rightarrow-\infty, \tilde{\sigma} \rightarrow-\infty$ if $\lambda \rightarrow \lambda_{1}^{\Omega}-$.

Proof of Lemma 9. If (1.9) admits a principal eigenvalue then any nonnegative associated eigenfunction $\phi \in H_{\Gamma^{\prime}}^{1}(\Omega)$ defines a strict weak supersolution to $-\Delta u-a u=0$. Thus, $\lambda_{1}(-a)>0$ (cf. [19]).

To prove the sufficiency of $\lambda_{1}(-a)>0$ we solve the variational problem $(2.20)$ by minimizing $I(u)=\int_{\Omega}\left\{|\nabla u|^{2}-a u^{2}\right\}-\int_{\Gamma} b u^{2}$ in $\tilde{\mathcal{M}}=\left\{u \in H_{\Gamma^{\prime}}^{1}(\Omega): \int_{\Gamma} u^{2}=1\right\}$. The key point is to show that $I$ is coercive on $\tilde{\mathcal{M}}$ and this precise fact follows from the fact that $\lambda_{1}(-a)$ is positive (see [17]). The issues of uniqueness, simplicity and continuous dependence on $a, b$ are shown in the same way as in Lemma 7 (cf. also [17]).

To obtain the divergence to $-\infty$ of the infimum $\tilde{\sigma}_{1}(a, b)$ when $\lambda_{1}(-a) \leq 0$ observe that a value $t_{0} \geq 0$ can be found so that $\lambda_{1}\left(-a+t_{0}\right)=0$. Since $\tilde{\sigma}_{1}(a, b) \leq \tilde{\sigma}_{1}\left(a-t_{0}, b\right)$ it suffices to show that $\tilde{\sigma}_{1}(a, b)=-\infty$ if $\lambda(-a)=0$. On the other hand, observe that if $\lambda_{1}(-a)=0$ then $\mu$ can be observed as an eigenvalue in (2.14). More precisely,

$$
\mu=\tilde{\sigma}_{1}(a-t, b)
$$

for $t>0$, since $\lambda_{1}(-a+t)>0$ for positive $t$. Now, $(2.16)$ can be read as

$$
\lim _{t \rightarrow 0+} \tilde{\sigma}_{1}(a-t, b)=-\infty .
$$

The conclusion then follows from the fact that $\tilde{\sigma}_{1}(a, b)<\tilde{\sigma}_{1}(a-t, b)$ for $t>0$.

Regarding (2.21), choose $\phi \in H_{\Gamma^{\prime}}^{1}(\Omega), \psi \in H_{\Gamma^{\prime}}^{1}(\Omega)$ positive eigenfunctions associated to $\sigma_{1}(a, b)$ and $\tilde{\sigma}_{1}(a, b)$, respectively. By employing $\psi$ as a test function in the weak eigenvalue equation for $\phi$ and the symmetric proceeding in the equation for $\psi$ it is obtained that

$$
\sigma_{1}(a, b) \int_{\Omega} \phi \psi=\tilde{\sigma}_{1}(a, b) \int_{\Gamma} \phi \psi
$$

Since $\phi, \psi$ are positive in $\Omega$ and $\phi \not \equiv 0$ on $\Gamma$ the desired sign equality is achieved.

Remark 3. Some of the features in Lemma 7 and Lemma 9, relevant for our subsequent work, are still valid if the conditions $a \in L^{\infty}(\Omega), b \in L^{\infty}(\Gamma)$ are replaced by measurable functions $a, b$ which are only (essentially) bounded above, assumed that $I(u)$ is finite at some $u \in H_{\Gamma^{\prime}}^{1}(\Omega)$.

First, by defining $\sigma_{1}(a, b)$ through the variational expression (1.6) then $\sigma_{1}(a, b)$ is finite if $I(u)$ finite at some $u \in H_{\Gamma^{\prime}}^{1}(\Omega)$. To prove that $I$ achieves the infimum at some (nonnegative) $\phi \in H_{\Gamma^{\prime}}^{1}(\Omega)$ it is shown as in Lemma 7 that $I$ is coercive in $\mathcal{M}$ while its weakly continuous character is a consequence of Fatou's Lemma.

Second, it is also checked that $\sigma_{1}(a, b)$ varies continuously with respect to perturbations of the form $a \rightarrow a \pm \varepsilon, b \rightarrow b \pm \varepsilon, \varepsilon>0$ a parameter. However, a stronger perturbation result holds. Namely,

$$
\sigma_{1}(a, b)=\lim \sigma_{1}\left(a_{n}, b_{n}\right)
$$


where $a_{n} \in L^{\infty}(\Omega), b_{n} \in L^{\infty}(\Gamma)$ are defined as $a_{n}=\max \{a,-n\}, b_{n}=\max \{b,-n\}$. In fact, by choosing positive eigenfunctions $\phi_{n} \in H_{\Gamma^{\prime}}^{1}(\Omega), \int_{\Omega} \phi_{n}^{2}=1$, associated to $\sigma_{1, n}:=\sigma_{1}\left(a_{n}, b_{n}\right)$ one finds that

$$
\sigma_{1, n}=\int_{\Omega}\left\{\left|\nabla \phi_{n}\right|^{2}-a_{n} \phi_{n}^{2}\right\}-\int_{\Gamma} b \phi_{n}^{2} \leq \sigma_{1}(a, b) .
$$

Now, (2.11) implies that $\phi_{n}$ is bounded in $H^{1}$ and, by passing through a subsequence, $\phi_{n} \rightarrow \phi_{0}$ weakly in $H^{1}$ and strongly both in $L^{2}(\Omega)$ and in $L^{2}(\Gamma)$. Taking inf-limits in (2.24) and Fatou's Lemma provide

$$
\int_{\Omega}\left\{\left|\nabla \phi_{0}\right|^{2}-a \phi_{0}^{2}\right\}-\int_{\Gamma} b \phi_{0}^{2} \leq \lim \sigma_{1, n} \leq \sigma_{1}(a, b)
$$

what proves (2.23).

Finally, the same facts hold for $\tilde{\sigma}_{1}(a, b)$ with $a, b$ bounded above provided that $\lambda_{1}(-a)>0$ (observe that $\lambda(-a)$ is well defined). In particular that $\tilde{\sigma}_{1}(a, b)=\lim \tilde{\sigma}_{1}\left(a_{n}, b_{n}\right)$, with an associated positive eigenfunctions sequence $\psi_{n}, \int_{\gamma} \psi_{n}^{2}=1$ and $\psi_{n} \rightarrow \psi_{0}$ weakly in $H^{1}$, with $\psi_{0}$ a nonnegative eigenfunction corresponding to $\tilde{\sigma}_{1}(a, b)$. After taking limits in $(2.22)$ with $a_{n}, b_{n}$ replacing $a, b$, we achieve

$$
\sigma_{1}(a, b) \int_{\Omega} \phi_{0} \psi_{0}=\tilde{\sigma}_{1}(a, b) \int_{\Gamma} \phi_{0} \psi_{0}
$$

Since $\phi_{0} \not \equiv 0$ on $\Gamma$ (by the same reasons as in Lemma 7) this implies that $\sigma_{1}(a, b)$ and $\tilde{\sigma}_{1}(a, b)$ possess the same sign. Notice that this inequality can not be directly obtained as in the case of bounded coefficients $a, b$ since $\phi_{0}, \psi_{0}$ are not now "a priori" weak solutions to the corresponding eigenvalue problems.

For its later application in Section 4, we need to consider problem (1.5) for a more general class of unbounded weights $a, b$ than those mentioned in Remark 3. In fact, notice that if $a(x)=\infty$ in, say, some open bounded part $D$ of $\Omega$ or $b(x)=\infty$ in an open subset $\Lambda \subset \Gamma$ then $\sigma_{1}(a, b)=-\infty$.

On the other hand, if $a(x)=-\infty$ in an open $D \subset \Omega, D \neq \Omega$ or if $b(x)=-\infty$ in a corresponding open set $\Lambda \subset \Gamma, \Lambda \neq \Gamma$ then $\sigma_{1}(a, b)$ can still be finite. This fact is precisely described in our next result where we are considering that $D=\Omega \backslash \bar{\Omega}_{0}, \Omega_{0} \subset \Omega$ a smooth subdomain, while $\Lambda \subset \Gamma$ is open and $\bar{\Gamma}$ constitutes a smooth manifold with boundary. It will be assumed in addition that $\partial \Omega_{0} \cap \Omega, \partial \Omega_{0} \cap \Gamma^{\prime}, \partial \Omega_{0} \cap \Lambda, \partial \Omega_{0} \cap \Gamma \backslash \bar{\Lambda}$ are all open in $\partial \Omega_{0}$ so that their closures define smooth manifolds with boundary, $\partial \Omega_{0}$ being the union of those closures.

In order to simplify the exposition we are fixing the notations $\Gamma_{0}:=\Gamma \backslash \bar{\Lambda}, \Gamma_{1}=\partial \Omega_{0} \cap$ $\left.\Gamma \backslash \bar{\Lambda}=\partial \Omega_{0} \cap \Gamma_{0}, \Gamma_{2}:=\partial \Omega_{0} \backslash \bar{\Gamma}_{1}\right)$ (Section 1).

Lemma 10. Suppose that $a, b$ are measurable functions defined in $\Omega$ and $\Gamma$, respectively, which are bounded from above and such that $a=-\infty$ in $D, b=-\infty$ on $\Lambda$. Then $\sigma_{1}(a, b)$, defined by (1.6), coincides with the principal eigenvalue of the problem

$$
\begin{cases}-\Delta u=a(x) u+\sigma u & \text { in } \Omega_{0}, \\ \frac{\partial u}{\partial \nu}=b(x) u & \text { on } \Gamma_{1}, \\ u=0 & \text { on } \Gamma_{2},\end{cases}
$$


provided $\Gamma_{1} \neq \emptyset$, or $\sigma_{1}(a, b)$ coincides with the first Dirichlet eigenvalue $\lambda_{1}^{\Omega_{0}}$ of $-\Delta-a$ in $\Omega_{0}$ if $\Gamma_{1}=\emptyset$.

Proof. We are assuming in what follows that the principal eigenvalue $\sigma_{1}^{\Omega_{0}}(a, b)$ of $(2.26)$ is finite (see Remark 3). We are also using the convention $0 \cdot \infty=0$ to handle integrals as $\int_{\Omega} a u^{2}, \int_{\Gamma} b u^{2}$ when $u$ vanishes in zones of $D$ or $\Lambda$ (an equivalent way of avoiding that singular product is to replace these integrals by $\left.\int_{\{u \neq 0\}} a u^{2}, \int_{\{u \neq 0\}} b u^{2}\right)$.

Assume that $\Gamma_{1} \neq \emptyset$, and observe that functions $u \in H_{\Gamma_{2}}^{1}\left(\Omega_{0}\right)$ can be extended by zero to $\Omega$, the resulting extensions verifying $\tilde{u} \in H_{\Gamma^{\prime}}^{1}(\Omega)$. By using such extensions in the variational characterization (1.6) of $\sigma_{1}(a, b)$ we obtain

$$
\sigma_{1}(a, b) \leq \sigma_{1}^{\Omega_{0}}(a, b)
$$

On the other hand, let $\left\{u_{n}\right\} \subset H_{\Gamma^{\prime}}^{1}(\Omega)$ be a minimizing sequence for $I$ in $\mathcal{M}=\left\{u \in H_{\Gamma^{\prime}}^{1}(\Omega)\right.$ : $\left.\int_{\Omega} u^{2}=1\right\}$, i. e. $I\left(u_{n}\right) \rightarrow \sigma_{1}(a, b)$. Since $\sigma_{1}(a, b)$ is finite, it follows that $I\left(u_{n}\right)$ is finite for large $n$, and thus $u_{n} \equiv 0$ in $D \cup \Lambda$. In view of the smoothness of $\Omega_{0}, \Lambda, \partial \Omega_{0} \cap \Omega, \partial \Omega_{0} \cap \Gamma^{\prime}$, $\partial \Omega_{0} \cap \Lambda$ and $\Gamma_{1}=\partial \Omega_{0} \cap \Gamma \backslash \bar{\Lambda}$, this implies that $u_{n} \in H_{\Gamma_{2}}^{1}\left(\Omega_{0}\right)$, and so $I\left(u_{n}\right) \geq \sigma_{1}^{\Omega_{0}}(a, b)$. Letting $n \rightarrow \infty$ we arrive at $\sigma_{1}(a, b) \geq \sigma_{1}^{\Omega_{0}}(a, b)$, which proves the lemma.

The case $\Gamma_{1}=\emptyset$ is handled in a similar manner.

\section{Remark 4.}

a) An alternative proof of Lemma 10 can be given by substituting $a, b$ by a sequence of bounded coefficients $a_{n}, b_{n}$ diverging to $-\infty$ in $D$ and $\Gamma$, respectively. Setting $a_{n}=$ $\max \{a,-n\}, b_{n}=\max \{b,-n\}\left(a_{n}, b_{n} \in L^{\infty}\right)$ we obtain $\sigma_{1}\left(a_{n}, b_{n}\right)$ increasing and,

$$
\sigma_{1}\left(a_{n}, b_{n}\right) \leq \sigma_{1}(a, b) \leq \sigma_{1}^{\Omega_{0}}(a, b)<\infty
$$

the last inequality being achieved as in Lemma 10. According to the ideas in Remark 3 one obtains that the normalized sequence of positive principal eigenfunctions $\phi_{n} \in H_{\Gamma^{\prime}}(\Omega)$ converges to some $\phi_{0} \in H_{\Gamma^{\prime}}^{1}(\Omega)$ weakly in $H^{1}$ and strongly in $L^{2}(\Omega), L^{2}(\Gamma)$. Thus, Fatou's Lemma provides

$$
\int_{\Omega}\left\{\left|\nabla \phi_{0}\right|^{2}-a \phi_{0}^{2}\right\}-\int_{\Gamma} b \phi_{0}^{2} \leq \sigma_{1}(a, b)<\infty
$$

together with $\phi_{0} \equiv 0$ both in $D$ and $\Gamma$. Since this implies $\phi_{0} \in H_{\Gamma_{2}}^{1}\left(\Omega_{0}\right)$ we obtain from the previous inequalities,

$$
\sigma_{1}^{\Omega_{0}}(a, b) \leq \int_{\Omega_{0}}\left\{\left|\nabla \phi_{0}\right|^{2}-a \phi_{0}^{2}\right\}-\int_{\Gamma_{1}} b \phi_{0}^{2} \leq \sigma_{1}(a, b) .
$$

This proves again Lemma 10 .

b) It can be shown (see the proof of Theorem 3 in Section 4) that the inequality $(2.27)$ is strict, i. e.,

$$
\sigma_{1}(a, b)<\sigma_{1}^{\Omega_{0}}(a, b)
$$




\section{Proof of Theorem 1}

This section is devoted to the proof of Theorem 1. For the sake of clarity, we divide it in several lemmas. The first one is concerned with the necessity of condition (1.8).

Lemma 11. Assume problem (1.2) admits a bounded positive weak solution $u \in H_{\Gamma^{\prime}}^{1}(\Omega) \cap$ $L^{\infty}(\Omega)$. Then

$$
\sigma_{1}\left(f_{\infty}, g_{\infty}\right)>0, \quad \text { and } \quad \sigma_{1}\left(f_{0}, g_{0}\right)<0 \text {. }
$$

Proof. We use the variational characterization of the eigenvalues (1.6). Take $u$ as a test function in (2.1), to obtain

$$
\int_{\Omega}|\nabla u|^{2}=\int_{\Omega} f(x, u) u+\int_{\Gamma} g(x, u) u<\int_{\Omega} f_{0}(x) u^{2}+\int_{\Gamma} g_{0}(x) u^{2} .
$$

Thus from (1.6) we have $\sigma_{1}\left(f_{0}, g_{0}\right)<0$.

On the other hand, let

$$
a(x)=\frac{f\left(x,|u|_{\infty}\right)}{|u|_{\infty}}, \quad b(x)=\frac{g\left(x,|u|_{\infty}\right)}{|u|_{\infty}}
$$

which are bounded measurable functions. Consider the eigenvalue problem (1.5). According to Lemma 7, the principal eigenvalue $\sigma_{1}(a, b)$ has an eigenfunction $\phi \in H_{\Gamma^{\prime}}^{1}(\Omega)$. By the definition of weak solution to (1.2) we have

$$
\int_{\Omega} \nabla u \nabla \phi=\int_{\Omega} f(x, u) \phi+\int_{\Gamma} g(x, u) \phi>\int_{\Omega} a(x) u \phi+\int_{\Gamma} b(x) u \phi
$$

On the other hand, since $\phi$ is an eigenfunction, it also follows that

$$
\int_{\Omega} \nabla \phi \nabla u=\int_{\Omega} a(x) \phi u+\int_{\Gamma} b(x) \phi u+\sigma_{1}(a, b) \int_{\Omega} \phi u
$$

Thus it is clear that $\sigma_{1}(a, b)>0$. Since $a>f_{\infty}, b>g_{\infty}$, it also follows that $\sigma_{1}\left(f_{\infty}, g_{\infty}\right)>0$, by the decreasing character of the principal eigenvalue to (1.5) with respect to the weights $a, b$.

We now prove that condition (1.8) implies that problem (1.2) has at least a positive weak solution which is additionally bounded (see Section 2). The proof is based on standard minimization of the functional associated to (1.2), which will be shown to be coercive. Before proceeding to the proof, we make an extension of $f$ and $g$ by letting $f(x, u)=f(x, 0)$ and $g(x, u)=g(x, 0)$ if $u \leq 0$.

Lemma 12. Assume $f$ and $g$ verify hypotheses $(\mathrm{H})$ and

$$
\sigma_{1}\left(f_{\infty}, g_{\infty}\right)>0, \quad \sigma_{1}\left(f_{0}, g_{0}\right)<0 .
$$

Then problem (1.2) admits at least a positive weak solution $u \in H_{\Gamma^{\prime}}^{1}(\Omega) \cap L^{\infty}(\Omega)$. 
Proof. We are proving that the natural functional $J$, whose critical points coincide with weak solutions to (1.2), is coercive and weakly sequentially lower semicontinuous. Thus, it will be standard to obtain a global minimizer of $J$. Let us begin by seeing that the functional

$$
J(u)=\frac{1}{2} \int_{\Omega}|\nabla u|^{2}-\int_{\Omega} F(x, u)-\int_{\Gamma} G(x, u),
$$

where $F(x, u)=\int_{0}^{u} f(x, s) d s, G(x, u)=\int_{0}^{u} g(x, s) d s$, is coercive in $H_{\Gamma^{\prime}}^{1}(\Omega)$.

Suppose not. Then there exists a sequence $\left\{u_{n}\right\} \subset H_{\Gamma^{\prime}}^{1}(\Omega)$ such that $\left|u_{n}\right|_{1} \rightarrow+\infty$, while $J\left(u_{n}\right) \leq C$, being $\left|u_{n}\right|_{1}^{2}=\int_{\Omega}\left|\nabla u_{n}\right|^{2}$ the equivalent norm involved. Let us show that this will lead to a contradiction. Set

$$
s_{n}^{2}=\int_{\Gamma} u_{n}^{2}, \quad t_{n}^{2}=\int_{\Omega} u_{n}^{2} .
$$

We claim that (up to a subsequence) $t_{n} \rightarrow+\infty$. Indeed, by assumption, it follows that (all the forthcoming constants will be renamed as $C$ )

$$
\frac{1}{2} \int_{\Omega}\left|\nabla u_{n}\right|^{2} \leq \int_{\Omega} F(x, u)+\int_{\Gamma} G(x, u)+C \leq C\left(1+s_{n}^{2}+t_{n}^{2}\right) .
$$

If we assume that $t_{n}$ is bounded, then $s_{n} \rightarrow+\infty$, so that putting $v_{n}=u_{n} / s_{n}$, we obtain

$$
\frac{1}{2} \int_{\Omega}\left|\nabla v_{n}\right|^{2} \leq C\left(\frac{1}{s_{n}^{2}}+1+\frac{t_{n}^{2}}{s_{n}^{2}}\right) \leq C .
$$

Thus there exists $v \in H_{\Gamma^{\prime}}^{1}(\Omega)$ such that $v_{n} \rightarrow v$ weakly in $H_{\Gamma^{\prime}}^{1}(\Omega)$ and strongly in $L^{2}(\Omega)$ and $L^{2}(\partial \Omega)$. However, this leads directly to a contradiction since then $v=0$ in $\Omega$ while $\int_{\Gamma} v^{2}=1$. Thus we may assume $t_{n} \rightarrow+\infty$. Let $w_{n}=u_{n} / t_{n}$. It follows as before that

$$
\frac{1}{2} \int_{\Omega}\left|\nabla w_{n}\right|^{2} \leq C\left(\frac{1}{t_{n}^{2}}+\frac{s_{n}^{2}}{t_{n}^{2}}+1\right)
$$

Thanks to Lemma 6, we have that

$$
\frac{s_{n}^{2}}{t_{n}^{2}}=\int_{\Gamma} w_{n}^{2} \leq \varepsilon \int_{\Omega}\left|\nabla w_{n}\right|^{2}+C(\varepsilon) \int_{\Omega} v_{n}^{2}=\varepsilon \int_{\Omega}\left|\nabla w_{n}\right|^{2}+C(\varepsilon) .
$$

Thus choosing and fixing $\varepsilon$ small enough, we get from (3.1) that

$$
\int_{\Omega}\left|\nabla w_{n}\right|^{2} \leq C\left(\frac{1}{t_{n}^{2}}+1\right)
$$

and we deduce again that, up to a subsequence, $w_{n} \rightarrow w$ weakly in $H^{1}(\Omega)$ and strongly in $L^{2}(\Omega)$ and $L^{2}(\partial \Omega)$. In particular, $\int_{\Omega} w^{2}=1$. Since

$$
\frac{1}{2} \int_{\Omega}\left|\nabla w_{n}\right|^{2} \leq \frac{C}{t_{n}^{2}}+\int_{\Omega} \frac{F\left(x, t_{n} w_{n}\right)}{t_{n}^{2}}+\int_{\Gamma} \frac{G\left(x, t_{n} w_{n}\right)}{t_{n}^{2}},
$$

we arrive at

$$
\frac{1}{2} \int_{\Omega}|\nabla w|^{2} \leq \limsup _{n \rightarrow+\infty} \int_{\Omega} \frac{F\left(x, t_{n} w_{n}\right)}{t_{n}^{2}}+\limsup _{n \rightarrow+\infty} \int_{\Gamma} \frac{G\left(x, t_{n} w_{n}\right)}{t_{n}^{2}} .
$$


However, it can be proved exactly as in [10] that this leads to

$$
\int_{\Omega}|\nabla w|^{2} \leq \int_{\Omega \cap\{w>0\}} f_{\infty} w^{2}+\int_{\Gamma \cap\{w>0\}} g_{\infty} w^{2}
$$

Since $\sigma_{1}\left(f_{\infty}, g_{\infty}\right)>0$, the variational characterization (1.6) of this eigenvalue implies that $w^{+} \equiv 0$, and thus $w \equiv 0$, contradicting $|w|_{L^{2}}=1$. Thus $J$ is coercive.

On the other hand the growth conditions (1.3) together with Fatou's lemma imply that the functional $J$ is also (weakly sequentially) lower semicontinuous. As a consequence we obtain that $J$ achieves its global minimum at a function $u \in H_{\Gamma^{\prime}}^{1}(\Omega)$. By replacing $u$ with $u^{+}$, and since $J\left(u^{+}\right) \leq J(u)$, it can be assumed that $u$ is nonnegative. Let us show that it is also nontrivial.

For this goal, we are proving that there exists $\phi \in H_{\Gamma^{\prime}}^{1}(\Omega)$ such that $J(\phi)<0$ what implies $u \not \equiv 0$. In fact, observe that the condition $-\infty \leq \sigma_{1}\left(f_{0}, g_{0}\right)<0$ and the variational characterization of this eigenvalue permit to find a nontrivial and nonnegative function $\psi \in H_{\Gamma^{\prime}}^{1}(\Omega) \cap L^{\infty}(\Omega),|\psi|_{\infty}=1$, such that

$$
\int_{\Omega}|\nabla \psi|^{2}-\int_{\Omega} f_{0} \psi^{2}-\int_{\Gamma} g_{0} \psi^{2}<0
$$

The estimates

$$
\frac{F(x, t \psi)}{t^{2}} \geq f(x, 1) \psi^{2}, \quad \frac{G(x, t \psi)}{t^{2}} \geq g(x, 1) \psi^{2},
$$

$0<t \leq 1$, a.e in $\Omega$ and $\partial \Omega$ respectively, and Fatou's lemma show that

$$
\limsup _{\varepsilon \rightarrow 0+} \frac{J(\varepsilon \psi)}{\varepsilon^{2}}=\int_{\Omega}|\nabla \psi|^{2}-\int_{\Omega} f_{0} \psi^{2}-\int_{\Gamma} g_{0} \psi^{2}<0
$$

so we can take $\phi=\varepsilon \psi$ for a small $\varepsilon$.

Although we have produced an absolute, non trivial and nonnegative minimizer $u \in$ $H_{\Gamma^{\prime}}^{1}(\Omega)$ for $J$ we can not still assert that $u$ provides a weak solution to problem (1.2) (see Section 2). A little more work has to be done and we are next showing that $u$ can be indeed chosen in $L^{\infty}$. To this objective and following [10] we introduce the approximate problems

$$
\begin{cases}-\Delta u=f_{k}(x, u) & \text { in } \quad \Omega, \\ \frac{\partial u}{\partial \nu}=g_{k}(x, u) & \text { on } \quad \Gamma \\ u=0 & \text { on } \Gamma^{\prime}\end{cases}
$$

where, for $k>0$ an integer, $f_{k}(x, u)=\max \{-k u, f(x, u)\}, g_{k}(x, u)=\max \{-k u, g(x, u)\}$ if $u \geq 0, f_{k}(x, u)=0, g_{k}(x, u)=0$ when $u<0$. It can be checked that $f_{k}, g_{k}$ satisfy the hypotheses $(\mathrm{H})$ and the more restrictive condition (2.2). Setting $f_{k, 0}, g_{k, 0}, f_{k, \infty}, g_{k, \infty}$ the resulting functions in (1.7) with $f_{k}, g_{k}$ replacing $f, g$ it follows that $f_{0} \leq f_{k, 0}, g_{0} \leq g_{k, 0}$, $f_{\infty} \leq f_{k, \infty}$ and $g_{\infty} \leq g_{k, \infty}$ for every $k$. Since $f, g$ satisfy (1.8) then $\sigma_{1}\left(f_{k, 0}, g_{k, 0}\right)<0$ for every $k$. On the other hand,

$$
f_{k, \infty}=\max \left\{f_{\infty},-k\right\}, \quad g_{k, \infty}=\max \left\{g_{\infty},-k\right\} .
$$


Since both $f_{\infty}, g_{\infty}$ are bounded above, the perturbation result (2.23) in Remark 3 permits asserting that $\sigma_{1}\left(f_{\infty}, g_{\infty}\right)=\lim \sigma_{1}\left(f_{k, \infty}, g_{k, \infty}\right)$. Thus, $\sigma_{1}\left(f_{k, \infty}, g_{k, \infty}\right)>0$ for $k$ large.

According to the preceding discussion, problem (3.2) exhibits a global nontrivial and nonnegative minimizer $u_{k} \in H_{\Gamma^{\prime}}^{1}(\Omega)$ for the associated functional $J_{k}(u)$,

$$
J_{k}(u)=\int_{\Omega} \frac{1}{2}|\nabla u|^{2}-F_{k}(x, u)-\int_{\Gamma} G_{k}(x, u)
$$

$F_{k}(x, u)=\int_{0}^{u} f_{k}(x, s) d s, G_{k}(x, u)=\int_{0}^{u} g_{k}(x, s) d s$. However, due to (2.2), $u_{k}$ now defines a weak solution to (3.2). In addition, Lemma 5 implies that $u_{k} \in L^{\infty}(\Omega)$. To conclude, we are checking that $\tilde{u}=\min \left\{u, u_{k}\right\}$, where $u \in H_{\Gamma^{\prime}}^{1}(\Omega)$ is the global minimizer constructed above, satisfies

$$
J(\tilde{u}) \leq J(u)
$$

Then, $\tilde{u} \in L^{\infty}(\Omega)$. Hence, since $\tilde{u}$ is also a global minimizer, it actually defines a genuine weak solution to (1.2). For the sake of completeness we are next proving (3.3). In fact, (3.3) is equivalent to

$$
\begin{aligned}
\int_{\left\{u_{k}<u\right\} \cap \Omega}\left\{\frac{1}{2}\left|\nabla u_{k}\right|^{2}-F\left(x, u_{k}\right)\right\}- & \int_{\left\{u_{k}<u\right\} \cap \Gamma} G\left(x, u_{k}\right) \leq \\
& \int_{\left\{u_{k}<u\right\} \cap \Omega}\left\{\frac{1}{2}|\nabla u|^{2}-F(x, u)\right\}-\int_{\left\{u_{k}<u\right\} \cap \Gamma} G(x, u) .
\end{aligned}
$$

Since $J_{k}\left(u_{k}\right) \leq J_{k}(v)$ for all $v \in H_{\Gamma^{\prime}}^{1}(\Omega)$, by using $v=\max \left\{u, u_{k}\right\}$ we arrive at

$$
\begin{aligned}
\int_{\left\{u_{k}<u\right\} \cap \Omega}\left\{\frac{1}{2}\left|\nabla u_{k}\right|^{2}-F_{k}\left(x, u_{k}\right)\right\} & -\int_{\left\{u_{k}<u\right\} \cap \Gamma} G_{k}\left(x, u_{k}\right) \leq \\
& \int_{\left\{u_{k}<u\right\} \cap \Omega}\left\{\frac{1}{2}|\nabla u|^{2}-F_{k}(x, u)\right\}-\int_{\left\{u_{k}<u\right\} \cap \Gamma} G_{k}(x, u) .
\end{aligned}
$$

Thus, the former inequality follows from the latter by observing that

$$
\begin{aligned}
\int_{\left\{u_{k}<u\right\} \cap \Omega}\left\{F(x, u)-F\left(x, u_{k}\right)\right\} & \leq \int_{\left\{u_{k}<u\right\} \cap \Omega}\left\{F_{k}(x, u)-F_{k}\left(x, u_{k}\right)\right\}, \\
\int_{\left\{u_{k}<u\right\} \cap \Gamma}\left\{G(x, u)-G\left(x, u_{k}\right)\right\} & \leq \int_{\left\{u_{k}<u\right\} \cap \Gamma}\left\{G_{k}(x, u)-G_{k}\left(x, u_{k}\right)\right\} .
\end{aligned}
$$

This completes the proof of (3.3).

To summarize, we have proved that problem (1.2) has a nonnegative nontrivial bounded weak solution $u$. As remarked earlier, $u \in W_{\text {loc }}^{2, p}(\Omega) \cap C_{\text {loc }}^{1, \alpha}(\Omega)$ for every $p>1$ and $\alpha \in(0,1)$. Thus the strong maximum principle implies $u>0$ in $\Omega$. This concludes the proof.

The uniqueness of solutions claimed in Theorem 1 is a consequence of the following Lemma (cf. [10] and Lemma 8 in [17]).

Lemma 13. Let $u, v \in H_{\Gamma^{\prime}}^{1}(\Omega)$ be two bounded positive weak solutions to problem (1.2), where $f(x, u), g(x, u)$ satisfy hypotheses $(\mathrm{H})$. Then $u=v$. 
Proof. If $u, v$ are positive solutions to (1.2) the basic key in this approach is using $\left(u^{2}-v^{2}\right) / u$ and $\left(v^{2}-u^{2}\right) / v$ as test functions. However we cannot conclude in our problem that $u / v$, $v / u$ are bounded (particularly near the interface $\gamma=\bar{\Gamma} \cap \overline{\Gamma^{\prime}}$ ). Thus we are modifying the test functions to be used according to [24]. For $\varepsilon>0$, the quotients $(u+\varepsilon) /(v+\varepsilon)$ and $(v+\varepsilon) /(u+\varepsilon)$ are bounded. This implies that both,

$$
\varphi_{\varepsilon}=\frac{(u+\varepsilon)^{2}-(v+\varepsilon)^{2}}{u+\varepsilon}, \quad \psi_{\varepsilon}=\frac{(v+\varepsilon)^{2}-(u+\varepsilon)^{2}}{v+\varepsilon},
$$

belong to $H_{\Gamma^{\prime}}^{1}(\Omega)$. It can be easily seen in addition that:

$$
\int_{\Omega} \nabla u \nabla \varphi_{\varepsilon}-\nabla v \nabla \psi_{\varepsilon}=\int_{\Omega}\left|\nabla u-\frac{u+\varepsilon}{v+\varepsilon} \nabla v\right|^{2}+\left|\nabla v-\frac{v+\varepsilon}{u+\varepsilon} \nabla u\right|^{2} .
$$

On the other hand,

$$
\int_{\Omega} \nabla u \nabla \varphi_{\varepsilon}-\nabla v \nabla \psi_{\varepsilon}=\int_{\Omega} f(x, u) \varphi_{\varepsilon}-f(x, v) \psi_{\varepsilon}+\int_{\Gamma} g(x, u) \varphi_{\varepsilon}-g(x, v) \psi_{\varepsilon} .
$$

Therefore we arrive at the inequality,

$$
\begin{aligned}
& \int_{\Omega}\left(\frac{f(x, u)}{u+\varepsilon}-\frac{f(x, v)}{v+\varepsilon}\right)\left((u+\varepsilon)^{2}-(v+\varepsilon)^{2}\right) \\
& \quad+\int_{\Gamma}\left(\frac{g(x, u)}{u+\varepsilon}-\frac{g(x, v)}{v+\varepsilon}\right)\left((u+\varepsilon)^{2}-(v+\varepsilon)^{2}\right) \geq 0 .
\end{aligned}
$$

Our intention is to pass to the limit as $\varepsilon \rightarrow 0$ by means of Fatou's Lemma. For this aim, we need to have an upper estimate of the integrands by an integrable majorant. Let us show that this is possible for the first integrand (a similar calculation holds for the second). Let $\Omega_{1}=$ $\{x \in \Omega: f(x, u(x)) \leq 0$ or $f(x, v(x)) \leq 0\}, \Omega_{2}=\{x \in \Omega: f(x, u(x))>0, f(x, v(x))>0\}$. If $x \in \Omega_{1}$, we can assume that $f(x, u(x)) \leq 0$. Then

$$
\begin{aligned}
& \left(\frac{f(x, u)}{u+\varepsilon}-\frac{f(x, v)}{v+\varepsilon}\right)\left((u+\varepsilon)^{2}-(v+\varepsilon)^{2}\right) \\
& \quad=\left(\frac{f(x, u)}{u}\left(\frac{u}{u+\varepsilon}-\frac{v}{v+\varepsilon}\right)+\left(\frac{f(x, u)}{u}-\frac{f(x, v)}{v}\right) \frac{v}{v+\varepsilon}\right)\left((u+\varepsilon)^{2}-(v+\varepsilon)^{2}\right) \leq 0,
\end{aligned}
$$

since $u /(u+\varepsilon)$ increases in $u$, while $f(x, u) / u$ is decreasing in $u$. The same inequality is obtained if $f(x, v(x)) \leq 0$. On the other hand, for $x \in \Omega_{2}$, and assuming $u(x) \geq v(x)$ :

$$
\left(\frac{f(x, u)}{u+\varepsilon}-\frac{f(x, v)}{v+\varepsilon}\right)\left((u+\varepsilon)^{2}-(v+\varepsilon)^{2}\right) \leq f(x, u)(u+\varepsilon) \leq C,
$$

while a similar inequality holds if $u(x)<v(x)$. Hence we have shown that the first integrand is majorized by an integrable function in $\Omega$, namely $C \chi_{\Omega_{2}}$, where $\chi_{\Omega_{2}}$ stands for the characteristic function of $\Omega_{2}$. As already remarked, the same thing happens for the second integrand, and we can use Fatou's Lemma to obtain from (3.4) that

$$
\int_{\Omega}\left(\frac{f(x, u)}{u}-\frac{f(x, v)}{v}\right)\left(u^{2}-v^{2}\right)+\int_{\Gamma_{1}}\left(\frac{g(x, u)}{u}-\frac{g(x, v)}{v}\right)\left(u^{2}-v^{2}\right) \geq 0 .
$$

Taking into account that $f(x, u) / u$ and $g(x, u) / u$ are decreasing, we arrive at $u=v$, as was to be proved. 
Proof of Corollary 2. It follows from hypotheses $(\mathrm{H})$ that $f_{0}, g_{0}$ are bounded from below measurable functions while $f_{\infty}, g_{\infty}$ are measurable and bounded from above. If $\sigma_{1}\left(f_{\infty}, g_{\infty}\right)>$ 0 then $\lambda_{1}\left(-f_{\infty}\right)$ is positive since $\sigma_{1}\left(f_{\infty}, g_{\infty}\right)<\lambda_{1}\left(-f_{\infty}\right)$. Hence, $\tilde{\sigma}_{1}\left(f_{\infty}, g_{\infty}\right)$ is well defined and positive (Remark 3). Conversely, $\lambda_{1}\left(-f_{\infty}\right)$ and $\tilde{\sigma}_{1}\left(f_{\infty}, g_{\infty}\right)$ positive imply, in view of $(2.25)$, that $\sigma_{1}\left(f_{\infty}, g_{\infty}\right)$ is also positive.

On the other hand, if one assumes $\tilde{\sigma}_{1}\left(f_{0}, g_{0}\right)<0$ (including the possible value $-\infty$ ) then the variational expression of $\sigma_{1}\left(f_{0}, g_{0}\right)$ immediately gives its negativity. If, on the contrary, $-\infty \leq \sigma_{1}\left(f_{0}, g_{0}\right)<0$ then a careful checking shows the existence of some $\phi \in H_{\Gamma^{\prime}}^{1}(\Omega)$ with the additional property of being nontrivial on $\Gamma$ such that $-\infty \leq \int_{\Omega}\left\{|\nabla \phi|^{2}-f_{0} \phi^{2}\right\}-\int_{\Gamma} g_{0} \phi^{2}<0$. This suffices to conclude that $-\infty \leq \tilde{\sigma}_{1}\left(f_{0}, g_{0}\right)<0$.

\section{Applications}

This section is devoted to the analysis of some applications to particular kind of nonlinearities in (1.2). Let us begin by considering logistic-type nonlinearities, which lead to problem (1.11):

$$
\begin{cases}-\Delta u=\lambda u-a(x) u^{p} & \text { in } \quad \Omega \\ \frac{\partial u}{\partial \nu}=\mu u-b(x) u^{q} & \text { on } \quad \Gamma \\ u=0 & \text { on } \Gamma^{\prime}\end{cases}
$$

where $\lambda, \mu \in \mathbb{R}, p, q>1$, and $a, b$ are continuous functions vanishing in $\Omega_{0} \subset \Omega$ and $\Gamma_{0} \subset \Gamma$, respectively, $\Omega_{0}, \Gamma_{0}$ satisfying the structure conditions specified in Sections 1, 2 (see the discussion before Lemma 10). Thus, the functions $f(x, u)=\lambda u-a(x) u^{p}$ and $g(x, u)=\mu u-b(x) u^{q}$ verify hypotheses $(\mathrm{H})$.

Proof of Theorem 3 and Corollary 4 . We first notice that the eigenvalues $\sigma(\mu), \tau(\mu)$ satisfy $\sigma(\mu)<\tau(\mu)$ for every $\mu$. In fact, their variational characterizations,

$$
\sigma(\mu)=\inf _{\substack{u \in H_{\Gamma^{\prime}}^{\prime}(\Omega) \\ u \neq 0}} \frac{\int_{\Omega}|\nabla u|^{2}-\mu \int_{\Gamma} u^{2}}{\int_{\Omega} u^{2}} \text { and } \tau(\mu)=\inf _{\substack{u \in H_{\Gamma_{2}}^{1}\left(\Omega_{0}\right) \\ u \neq 0}} \frac{\int_{\Omega_{0}}|\nabla u|^{2}-\mu \int_{\Gamma_{0}} u^{2}}{\int_{\Omega_{0}} u^{2}},
$$

yield $\sigma(\mu) \leq \tau(\mu)$ since functions in $H_{\Gamma_{2}}^{1}\left(\Omega_{0}\right)$, when extended by zero outside $\Omega_{0}$, belong to $H_{\Gamma^{\prime}}^{1}(\Omega)$. Thus, the second infimum is taken in a smaller set. However, the equality $\sigma(\mu)=\tau(\mu)$ never occurs. Otherwise, a positive eigenfunction $\psi$ associated to $\tau$ and extended as zero to $\Omega$ would define a nonnegative eigenfunction $\phi$ to $\sigma$ which vanishes identically in $\Omega \backslash \bar{\Omega}_{0}$. That is not possible (see the preliminaries of Section 2).

To prove the theorem we only need to compute the eigenvalues $\sigma_{1}\left(f_{0}, g_{0}\right)$ and $\sigma_{1}\left(f_{\infty}, g_{\infty}\right)$ in this particular case and checking conditions (1.8). We have $f_{0}(x)=\lambda, g_{0}(x)=\mu$, and it is not hard to see that this entails $\sigma_{1}\left(f_{0}, g_{0}\right)=\sigma(\mu)-\lambda$. On the other hand,

$$
f_{\infty}(x)=\left\{\begin{array}{cl}
\lambda & \text { in } \Omega_{0} \\
-\infty & \text { in } D
\end{array} \quad g_{\infty}(x)=\left\{\begin{array}{cl}
\mu & \text { in } \Gamma_{0} \\
-\infty & \text { in } \Gamma \backslash \Gamma_{0}
\end{array}\right.\right.
$$


and thanks to Lemma 10, we obtain that $\sigma_{1}\left(f_{\infty}, g_{\infty}\right)=\tau(\mu)-\lambda$. A final application of Theorem 1 concludes the proof.

As for Corollary 4 notice that $\tilde{\sigma}_{1}\left(f_{0}, g_{0}\right)=\tilde{\sigma}(\lambda)-\mu$ while $\lambda_{1}\left(-f_{\infty}\right)=\lambda_{1}^{\Omega_{0}}-\lambda$ with $\tilde{\sigma}_{1}\left(f_{\infty}, g_{\infty}\right)=\tilde{\tau}(\lambda)-\mu$ (see notations in Section 1 and Remark $\left.2 \mathrm{~b}\right)$ ). Thus, it suffices to apply Corollary 2 .

As a second application consider the inclusion of weight functions $w, m \in L^{\infty}$ modulating the linear terms carrying the parameters in (1.11). Namely, the problem

$$
\begin{cases}-\Delta u=\lambda w(x) u-a(x) u^{p} & \text { in } \quad \Omega, \\ \frac{\partial u}{\partial \nu}=\mu m(x) u-b(x) u^{q} & \text { on } \Gamma, \\ u=0 & \text { on } \Gamma^{\prime}\end{cases}
$$

where $p, q>1, \lambda, \mu \in \mathbb{R}$, the coefficients $a, b$ satisfy the hypotheses quoted before Theorem 3 and $w, m$ are not restricted in sign. Problem (4.1) was treated in [11] under significantly more restrictive conditions: the components $\Gamma$ and $\Gamma^{\prime}$ must be separated $\left(\bar{\Gamma} \cap \bar{\Gamma}^{\prime}=\emptyset\right)$, the null set $\bar{\Omega}_{0}$ for $a$ can only meet the flux component $\Gamma$ in a whole connected piece, the positivity of $b$ on $\Gamma\left(\Gamma_{0}=\emptyset\right)$ and the presence of $\lambda$ as a single parameter $(\mu=1)$. The very particular case $w=0, a=0, m=1, \Gamma$ and $\Gamma^{\prime}$ separated components and also $b>0$ on $\Gamma$ was also dealt with in [25]. In addition, (4.1) was treated in [16] with $\Gamma^{\prime}=\emptyset$ (a single Robin condition on $\partial \Omega), b(x) \equiv 0, \mu=1, m \leq 0$, and more importantly, the restriction $\Omega_{0} \cap \partial \Omega=\emptyset$.

All the results just mentioned are improved as follows. Denoting by $\sigma_{1}(\lambda, \mu)$ and $\sigma_{1}^{\Omega_{0}}(\lambda, \mu)$ the principal eigenvalues of the problems

$$
\begin{cases}-\Delta u=\lambda w(x) u+\sigma u & \text { in } \quad \Omega, \\ \frac{\partial u}{\partial \nu}=\mu m(x) u & \text { on } \Gamma, \\ u=0 & \text { on } \Gamma^{\prime},\end{cases}
$$

and

$$
\begin{cases}-\Delta u=\lambda w(x) u+\sigma u & \text { in } \Omega_{0}, \\ \frac{\partial u}{\partial \nu}=\mu m(x) u & \text { on } \Gamma_{1}, \\ u=0 & \text { on } \Gamma_{2},\end{cases}
$$

respectively, we can use Theorem 1 to conclude that problem (4.1) exhibits a unique positive solution if and only if either

$$
\sigma_{1}(\lambda, \mu)<0<\sigma_{1}^{\Omega_{0}}(\lambda, \mu)
$$

if $\Gamma_{1} \neq \emptyset$, or

$$
\sigma_{1}(\lambda, \mu)<0<\lambda_{1}^{\Omega_{0}}(-\lambda w),
$$

provided $\Gamma_{1}=\emptyset$. It only remains to ensure that the $\lambda-\mu$ regions defined by (4.2) or (4.3) are always nonempty regardless the structure of the weights $w, m$ (this discussion is missing in [11]). We are proceeding with elementary methods while a more ambitious analysis is left for future reporting.

To this aim we are fixing the notation $\mathcal{C}_{0}=\left\{\sigma_{1}^{\Omega_{0}}(\lambda, \mu)>0\right\}$ and $\mathcal{C}=\left\{\sigma_{1}(\lambda, \mu)>0\right\}$. Observe that by employing the first part of the proof of Lemma 8 it follows that $\sigma_{1}(\lambda, \mu)$ 

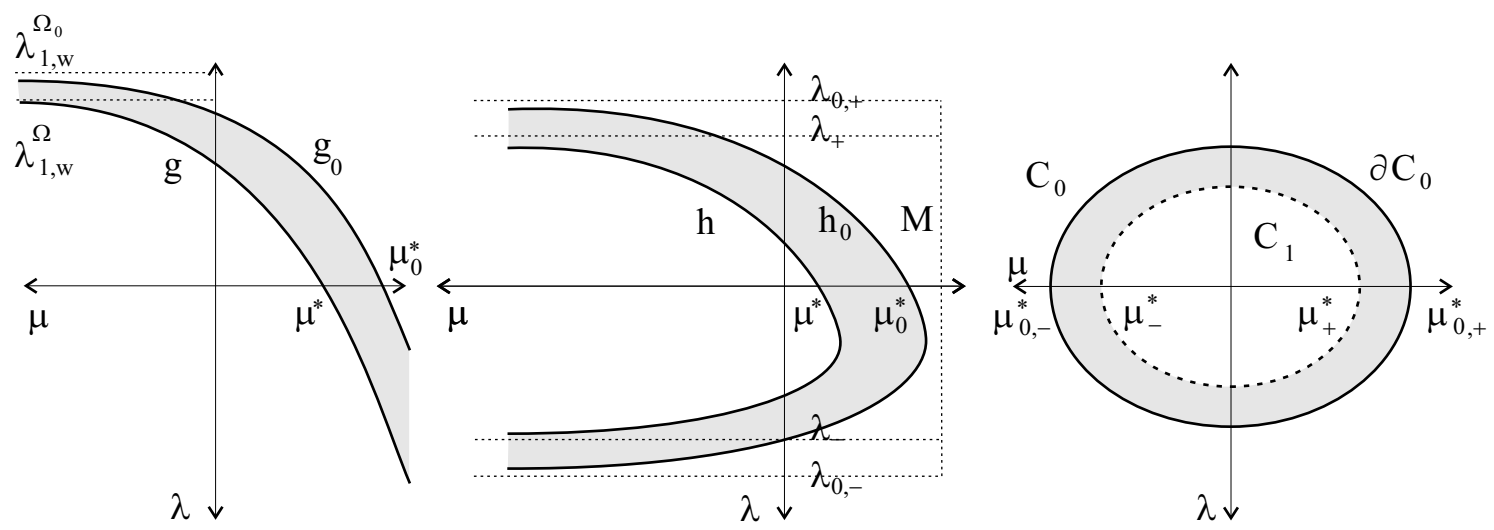

Figure 3: Existence regions (shadowed) corresponding to $\Gamma_{1} \neq \emptyset$. Left to right: 1) $m \geq 0$, $w \geq 0,2) m \geq 0, w$ both signs, 3) $m, w$ having both signs.

and $\sigma_{1}^{\Omega_{0}}(\lambda, \mu)$ are also concave in $(\lambda, \mu)$. Thus, $\mathcal{C}$ and $\mathcal{C}_{0}$ are convex. Recall also that $\sigma_{1}(\lambda, \mu)<\sigma_{1}^{\Omega_{0}}(\lambda, \mu)\left(\right.$ Remark 4). Thus $\overline{\mathcal{C}} \subset \mathcal{C}_{0}$.

Let us consider (4.2) and suppose that $m>0$ a. e. in $\Omega$. If, say, $w>0$ a. e. in $\Omega$ then $\sigma_{1}^{\Omega_{0}}(\lambda, \mu)$ is concave and separately decreasing in $\lambda, \mu$ together with $\sigma_{1}^{\Omega_{0}} \rightarrow \pm \infty$ as $\lambda \rightarrow \mp \infty$ while $\sigma_{1}^{\Omega_{0}} \rightarrow-\infty$ as $\mu \rightarrow \infty$ and $\sigma_{1}^{\Omega_{0}} \rightarrow \lambda_{1}^{\Omega_{0}}(-\lambda w)$ as $\mu \rightarrow-\infty$. This implies that $\sigma_{1}^{\Omega_{0}}=0$ consists of points $\lambda=g_{0}(\mu)$ for a certain decreasing continuous function $g_{0}$ such that $g_{0} \rightarrow-\infty$ as $\mu \rightarrow+\infty$ and $g_{0} \rightarrow \lambda_{1, w}^{\Omega_{0}}$ as $\mu \rightarrow-\infty$, where $\lambda=\lambda_{1, w}^{\Omega_{0}}$ stands for the principal eigenvalue to the weighted eigenvalue problem,

$$
\left\{\begin{array}{cl}
-\Delta u=\lambda w(x) u & x \in \Omega_{0} \\
u=0 & x \in \partial \Omega_{0} .
\end{array}\right.
$$

Similar properties of $\sigma_{1}(\lambda, \mu)$ imply that it defines a corresponding zero set $\lambda=g(\mu), g$ possessing the same properties as $g_{0}$ but now $g(\mu) \rightarrow \lambda_{1, w}^{\Omega}$ as $\mu \rightarrow-\infty$, where $\lambda=\lambda_{1, w}^{\Omega}$ is the principal eigenvalue to problem (4.4) observed instead in the domain $\Omega$. Moreover, since $\sigma_{1}(\lambda, \mu)<\sigma_{1}^{\Omega_{0}}(\lambda, \mu)$ then $g<g_{0}$ and the existence region $\mathcal{E}$ defined by (4.2) can be represented as $\mathcal{E}=\left\{g(\mu)<\lambda<g_{0}(\mu)\right\}$ (Figure 3). Exactly the same conclusions hold true if $m \neq 0, w \neq 0$ and their null sets can be represented as $\{m=0\}=\bar{\Omega}_{0, m},\{w=0\}=\bar{\Omega}_{0, w}$ with $\Omega_{0, m} \subset \Omega, \Omega_{0, w} \subset \Omega$ smooth domains with the same structure properties as $\Omega_{0}$ in Theorem 1 (Sections 1 and 2).

Let us go a step further and suppose $m>0$ a. e. in $\Omega$ while $w$ exhibits both signs in a nontrivial way, say $w>0$ and $w<0$ a. e. in certain balls $B_{+} \subset \Omega, B_{-} \subset \Omega_{-}$, respectively, which, to simplify the exposition, will be assumed to lie in $\Omega_{0}$. Observe now that for each $\mu$, $\sigma_{1}^{\Omega_{0}}(\lambda, \mu)$ is concave in $\lambda$ and $\sigma_{1}^{\Omega_{0}}(\lambda, \mu) \rightarrow-\infty$ as $\lambda \rightarrow \pm \infty$, while $\sigma_{1}^{\Omega_{0}}(\lambda, \mu)$ is still decreasing in $\mu$ for $\lambda$ fixed with the limit behaviour as $\mu \rightarrow \pm \infty$ stated above. Moreover, $\lambda_{1}^{\Omega_{0}}(-\lambda w)$ as a function of $\lambda$ has exactly two zeros $\lambda_{0,-}<0<\lambda_{0,+}$, the principal eigenvalues of (4.4), being positive in $\lambda_{0,-}<\lambda<\lambda_{0,+}$ and negative outside the closed interval $\lambda_{0,-} \leq \lambda \leq \lambda_{0,+}$. Thus $\mathcal{C}_{0}$ lies in the strip $\left\{\lambda_{0,-}<\lambda<\lambda_{0,+}\right\}$ and by using the variational characterization of $\sigma_{1}^{\Omega_{0}}$ one checks that $\sigma_{1}^{\Omega_{0}}(\lambda, \mu)<0$ in that strip provided $\mu \geq M$ for certain $M>0$ (Figure 3 ). In addition, $\sigma_{1}^{\Omega_{0}}(0, \mu)$ has the same sign as $\left(\mu_{0}^{*}-\mu\right), \mu_{0}^{*}>0$ the unique zero of $\sigma_{1}^{\Omega_{0}}(0, \mu)$. By using the concavity and continuity of $\sigma_{1}^{\Omega_{0}}(0, \mu)$ it follows the existence of a locally Lipschitz 
function $h_{0}(\lambda)$ with $h_{0} \rightarrow-\infty$ both as $\lambda \rightarrow \lambda_{0,-}+$ and $\lambda \rightarrow \lambda_{0,+}-$, sup $h_{0} \geq \mu_{0}^{*}$ and such that $\mathcal{C}_{0}=\left\{\mu<h_{0}(\lambda)\right\}$. Furthermore, by the decreasing character of $\sigma_{1}^{\Omega_{0}}$ in $\mu$, one can shows the existence of $\lambda_{0,-}<c_{1} \leq c_{2}<\lambda<\lambda_{0,+}$ with $h_{0}$ increasing for $\lambda \leq c_{1}, h_{0}$ decreasing in $\lambda \geq c_{2}$ while $h_{0}=\sup h_{0}$ in $c_{1} \leq \lambda \leq c_{2}$ ( $c_{1}$ has been chosen equal to $c_{2}$ in Figure 3 for simplicity). On the other hand, the same analysis reveals that $\mathcal{C}=\{\mu<h(\lambda)\}$ for a function $h$ with identical properties as $h_{0}$ but defined now in $\lambda_{-}<\lambda<\lambda_{+}$where $\lambda_{0_{-}-}<\lambda_{-}<0<\lambda_{+}<\lambda_{0,+}$ are the zeros of $\lambda_{1}^{\Omega}(-\lambda w)$, while $h(\mu)<h_{0}(\mu)$ for every $\lambda$. As a main conclusion, the existence region $\mathcal{E}$ defined by (4.2) consists in the set (see Figure 3) $\mathcal{E}=\left\{h(\lambda)<\mu<h_{0}(\lambda)\right\}$ where for those $\lambda_{0,-}<\lambda<\lambda_{0+}$ not lying in $\left[\lambda_{-}, \lambda_{+}\right]$it is understood that points with $\mu<h_{0}(\lambda)$ also belong to $\mathcal{E}$.

Suppose next that both $m$ and $w$ are two-signed in a nontrivial way. Consider also for simplicity that such signs are achieved in $\Gamma_{1}$ and $\Omega_{0}$, respectively. It follows from the variational characterization of $\sigma_{1}^{\Omega_{0}}$ that $\mathcal{C}_{0}$ is bounded. In fact, boundedness in $\lambda$ is achieved by choosing test functions compactly supported in balls in $\Omega_{0}$ where $w$ keeps its sign. After that, an election of test functions whose restriction to $\Gamma_{1}$ are compactly supported where $m$ has a fixed sign shows that $\mathcal{C}_{0}$ is bounded. In particular the same holds for $\mathcal{C}$. Moreover, since $\mathcal{C}_{0}$ is the positive level set of $\sigma_{1}^{\Omega_{0}}(\lambda, \mu)$, a continuous and concave function, then its boundary $\partial \mathcal{C}_{0}$ consists in a closed Lipschitz curve which surrounds $(0,0)$. Therefore, the existence region (4.2), i. e. $\mathcal{E}=\mathcal{C}_{0} \backslash \mathcal{C}_{1}, \mathcal{C}_{1}=\left\{\sigma_{1}^{\Omega_{0}}(\lambda, \mu) \geq 0\right\} \supset \mathcal{C}$, defines an annular open region that surrounds $(0,0)$ (notice that $\mathcal{C}_{1}$ is convex) and that has $\partial \mathcal{C}_{0}$ as a component of its boundary. Thus, the existence set $\mathcal{E}$ defines an isola of solutions (Figure 3 ).

Finally, observe that the analysis of all of the remaining cases corresponding to (4.2) $\left(\Gamma_{1} \neq \emptyset\right)$ and associated to different combinations of sign in $m$ and $w$ can be deduced from the previous analysis by merely changing $\lambda \rightarrow \pm \lambda, \mu \rightarrow \pm \mu$ in the functions $\sigma_{1}(\lambda, \mu)$, $\sigma_{1}^{\Omega_{0}}(\lambda, \mu)$. On the other hand, the discussion of the case $(4.3)\left(\Gamma_{1}=\emptyset\right)$, being much simpler, is omitted.

Another application of our results is furnished by the class of nonlinear reaction-diffusion problems

$$
\begin{cases}-\Delta u=\lambda u-a(x) u^{p} & \text { in } \quad \Omega, \\ \frac{\partial u}{\partial \nu}=\frac{\mu u}{1+k u}-b(x) u^{q} & \text { on } \Gamma, \\ u=0 & \text { on } \Gamma^{\prime},\end{cases}
$$

where $p, q>1, k$ is a positive constant, coefficients $a, b$ satisfy the structure conditions of Sections 1, 2 and $\lambda, \mu$ are regarded as parameters. Problem (4.5) can be regarded as a model for two couples of parallel reactions occurring both in a medium $\Omega$ and in an specific area $\Gamma$ of its boundary $\partial \Omega$, being the remaining part $\Gamma^{\prime}$ of $\partial \Omega$ inactive. Such reactions compite for the same product ( $u$ designating its concentration) and the whole process is subjected to diffusion. Both in $\Omega$ and on $\Gamma$ the consumption of $u$ is switched off in $\Omega_{0}$ and $\Gamma_{1}$, respectively. As a main difference with respect to (1.11) and (4.1), it is assumed that the production of $u$ in $\Gamma$ proceeds according to a Michaelis-Menten type law (saturation at large values of $u$ ).

Special cases of (4.5) have been considered in the literature. A one dimensional version with $a=b=0, \lambda=0, \mu=1$ is studied in [27] by perturbation methods. The $n$-dimensional case with $a=b=0, \lambda>0, \Gamma, \Gamma^{\prime}$ separated components ( $\Gamma^{\prime}$ supporting a Neumann condition) is analyzed in [26] by the method of sub an super solutions ([30] deals with the same case 
but $\Gamma^{\prime}=\emptyset$ and a nonnegative $f(x)$ perturbs the equation).

Under the light of Theorem 1, problem (4.5) has a unique positive solution if and only if,

$$
\sigma(\mu)<\lambda<\sigma_{1}^{*}\left(\Omega_{0}\right) \quad \mu \in \mathbb{R},
$$

provided $\Gamma_{1} \neq \emptyset$ or

$$
\sigma(\mu)<\lambda<\lambda_{1}^{\Omega_{0}} \quad \mu \in \mathbb{R}
$$

when $\Gamma_{1} \neq \emptyset$, being $\sigma(\mu)=\sigma_{1}(0, \mu)$ (see Theorem 3 ) and $\sigma_{1}^{*}\left(\Omega_{0}\right)=\sigma_{1}^{\Omega_{0}}(0,0)>0$.

Remarks 5. The bifurcation behavior with respect to $\lambda$ and $\mu$, of the positive solutions to the problems (1.11), (4.1) and (4.5) has not been discussed here by the sake of brevity. To outline the main features, it can be shown in the case of (1.11), for instance, that positive solutions bifurcate from zero at the curve $\lambda=\sigma(\mu)$ (Figure 1), while they bifurcate from infinity at the curve $\lambda=\tau(\mu)$ if $\Gamma_{1} \neq \emptyset$, and at $\lambda=\lambda_{1}^{\Omega_{0}}$ when $\Gamma_{1}=\emptyset$ (see [18], 8] and [31] for bifurcation phenomena generated by the boundary conditions).

\section{REFERENCES}

[1] B. Abdellaoui, E. Colorado, I. Peral, Some remarks on elliptic equations with singular potential and mixed boundary conditions, Adv. Nonl. Stud. 4 (2004), 503-533.

[2] B. Adbellaoui, E. Colorado, I. Peral, Existence and nonexistence results for a class of parabolic equations with mixed boundary conditions, Comm. Pure Appl. Anal. 5 (2006), 29-54.

[3] B. Adbellaoui, E. Colorado, I. Peral, Some critial elliptic problems with mixed Dirichlet-Neumann boundary conditions related with Sobolev and Hardy-Sobolev constants, preprint.

[4] R. A. Adams, Sobolev spaces. Pure and applied mathematics, vol. 65. Academic Press. New York-London, 1975.

[5] H. Amann, On the existence of positive solutions of nonlinear elliptic boundary value problems, Indiana Univ. Math. J. 21 (1971/72), 125-146.

[6] H. Amann, Nonlinear elliptic equations with nonlinear boundary conditions in "New developments in differential equations" (W. Eckhaus ed.), pp. 43-63. North-Holland Math. Studies, Vol. 21, North-Holland, Amsterdam, 1976.

[7] H. Amann, Maximum principles and principal eigenvalues, in "Ten mathematical essays on approximation in analysis and topology", 1-60, Elsevier B. V., Amsterdam, 2005.

[8] J. Arrieta, R. Pardo, A. Rodríguez-Bernal, Bifurcation and stability of equilibria with asymptotically linear boundary conditions at infinity, to appear in Proc. Roy. Soc. Edinburgh.

[9] H. Beirão DA Veiga, On the $W^{2, p}$-regularity for solutions of mixed problems, J. Math. Pures Appl. 53 (1974), 279-290. 
[10] H. Brézis, L. Oswald, Remarks on sublinear elliptic equations, Nonlinear Anal. 10 (1983), 55-64.

[11] S. Cano-Casanova, On the positive solutions of the logistic weighted elliptic BVP with sublinear mixed boundary conditions, in "Spectral theory and nonlinear analysis with applications to spatial ecology", S. Cano-Casanova, J. López-Gómez, C. MoraCorral eds. World Scientific Publishing co., Singapore, 2005.

[12] S. Cano-Casanova, J. López-Gómez, Properties of the principal eigenvalues of a general class of non-classical mixed boundary value problems, J. Differential Equations 178 (2002), 123-211.

[13] E. Colorado, I. Peral, Semilinear elliptic problems with mixed Dirichlet-Neumann boundary conditions, J. Funct. Anal. 199 (2003), 468-507.

[14] E. Colorado, I. Peral, Some results for elliptic eigenvalue problems with moving mixed boundary conditions, Equadiff 2003, 540-545, World Sci. Publ., Hackensack, NJ, 2005.

[15] E. Colorado, I. Peral, Eigenvalues and bifurcation for elliptic equations with mixed Dirichlet-Neumann boundary conditions related to Caffarelli-Kohn-Nirenberg inequalities, Topol. Meth. Nonl. Anal. 23 (2004), 239-273.

[16] J. M. Fraile, P. Koch-Medina, J. López-Gómez, S. Merino, Elliptic eigenvalue problems and unbounded continua of positive solutions of a semilinear elliptic equation, J. Differential Equations 127 (1996), 295-319.

[17] J. García-Melián, J. D. Rossi, J. Sabina de Lis, A bifurcation problem governed by the boundary condition I, to appear in NoDEA Nonlinear Differ. Equ. Appl.

[18] J. García-Melián, J. D. Rossi, J. Sabina de Lis, A bifurcation problem governed by the boundary condition II, to appear in Proc. London Math. Soc.

[19] J. García-Melián, J. Sabina DE Lis, Maximum and comparison principles for operators involving the p-Laplacian, J. Math. Anal. Appl. 218 (1998), 49-65.

[20] D. Gilbarg, N. Trudinger, Elliptic partial differential equations of second order, Springer-Verlag, Berlin, 1983.

[21] A. A. Lacey, J. R. Ockendon, J. SAbina, Multidimensional reaction diffusion equations with nonlinear boundary conditions, SIAM J. Appl. Math. 58 (1998), 16221647.

[22] G. Lieberman, Mixed boundary value problems for elliptic and parabolic differential equations of second order, J. Math. Anal. Appl. 113 (1986), 422-440.

[23] G. Lieberman, Optimal Hölder regularity for mixed boundary value problems, J. Math. Anal. Appl. 143 (1989), 572-586. 
[24] P. Lindqvist, On the equation $\operatorname{div}\left(|\nabla u|^{p-2} \nabla u\right)+\lambda|u|^{p-2} u=0$, Proc. Amer. Math. Soc. 109 (1990), 157-164.

[25] C. Morales-Rodrigo, A. SuÁrez, Some elliptic problems with nonlinear boundary conditions, in "Spectral theory and nonlinear analysis with applications to spatial ecology", S. Cano-Casanova, J. López-Gómez, C. Mora-Corral eds. World Scientific Publishing co., Singapore, 2005.

[26] C. V. PAO, Nonlinear Parabolic and Elliptic Equations, Plenum Press, New York, 1992.

[27] L. W. Ross, Perturbation analysis of diffusion-coupled biochemical reaction kinetics, SIAM J. Appl. Math. 19 (1970), 323-329.

[28] J. D. Rossi, Elliptic problems with nonlinear boundary conditions and the Sobolev trace theorem. Stationary partial differential equations. Vol. II, 311-406, Handbook on Differential Equations, Elsevier/North-Holland, Amsterdam, 2005.

[29] M. Struwe, Variational Methods, Springer Verlag, Heidelberg, New York, 1989.

[30] K. Umezu, Nonlinear elliptic boundary value problems suggested by fermentation, NoDEA Nonlinear Differ. Equ. Appl. 7 (2000), 143-155.

[31] K. UMEZU, Global positive solution branches of positone problems with nonlinear boundary conditions, Differential and Integral Equations 13 (2000), 669-686. 